\title{
Optimization of frequency domain impedances for time-domain response analyses of building structures with rigid shallow foundations
}

Earthquake Spectra 2021, Vol. 37(3) 1955-1979 (C) The Author(s) 2021

Article reuse guidelines: sagepub.com/journals-permissions DOI: I0.1 I77/875529302098|994

journals.sagepub.com/home/eqs

\author{
Danilo S Kusanovic' \\ Elnaz Seylabi, M.EERI ${ }^{2}$, \\ and Domniki Asimaki, M.EERI'
}

\begin{abstract}
The effects of dynamic soil-structure interaction (SSI) have been extensively studied in the last few decades, and proper analysis for the linear elastic case in frequency domain has been established successfully. However, SSI is rarely considered in the design of building structures, and instead, buildings are frequently analyzed using a rigid base assumption and quasi-static loading conditions that ignore SSI and its dynamic nature. Acknowledging these shortcomings, the National Institute of Standards and Technology (NIST) published in 2012 a set of recommendations on time-domain analyses of SSI for building structures compatible with standard finite element packages for consideration in engineering design. The so-called NIST GCR |2-9|7-2| report introduced a major simplification to enable frequency domain tools to be implemented in time domain analyses. That is, replacing the frequencydependent soil impedance functions by a single-valued functions read at the flexiblebase structure frequency; This work seeks to quantify the accuracy of this simplification considering fully coupled two-dimensional (2D) finite element models (FEM) as the reference. Using a Bayesian approach based on ensemble Kalman inversion (EnKI) and a range of numerical simulations of soil-foundation-building interaction, we estimate the optimal frequency that can be used to estimate soil impedance for time domain analyses; and we evaluate the improvement that the corresponding impedance offers relative to the full FEM results when compared to time domain analyses performed in accordance to the NIST recommendations outlined above.
\end{abstract}

\footnotetext{
'Department of Mechanical and Civil Engineering, California Institute of Technology, Pasadena, CA, USA

${ }^{2}$ Department of Civil and Environmental Engineering, University of Nevada, Reno, Reno, NV, USA
}

\section{Corresponding author:}

Domniki Asimaki, Department of Mechanical and Civil Engineering, California Institute of Technology, 34I GatesThomas Building, Pasadena, CA 9I 125, USA.

Email: domniki@caltech.edu 


\section{Keywords \\ Soil-structure interaction, system identification, ensemble Kalman inversion, period elongation, radiation damping, inverse problem, NIST, reduced order model, mathe- matical modeling}

Date received: 5 April 2020; accepted: 2 I November 2020

\section{Introduction}

Accurate quantification of dynamic soil-structure interaction (SSI) effects is critical in the design of earthquake-resistant structures. Poorly estimated or neglected SSI effects have been shown to yield unsafe or overly conservative designs, depending on the resulting over- or under-estimation of the critical response of the structure (Mynolakis and Gazetas, 2000; Veletsos and Nair, 1975). In general, SSI analyses are carried out using two families of methods, namely the direct and the substructure method (Bathe, 2006; Hughes, 2012; Wolf, 1985, 1989; Wolf and Obernhuber, 1985). In the direct method, the structure and the near-field soil are modeled explicitly, while artificial boundary conditions, which ideally introduce transparency to incoming and outgoing waves, are introduced to truncate the semi-infinite extent of the far-field soil (Basu and Chopra, 2003; Kucukcoban and Kallivokas, 2011; Lysmer et al., 1969).

The substructure method (Bielak, 1974; Parmelee, 1967; Wolf and Obernhuber, 1985), on the other hand, divides the SSI problem into two sub-systems: the structure and the soil subsystem, which is represented by impedance functions (Gazetas, 1991; Pais and Kausel, 1988; Seylabi et al., 2016; Veletsos and Meek, 1974). These functions are complex-valued, the real part is the representation of the soil stiffness and inertia while the imaginary part is the representation of geometric damping within the soil. To excite the model more realistically, one needs to modify the free field motion (FFM) to map the coherency effects introduced by the differential compliance of the foundation and the surrounding soil. Despite the computational efficiency of the substructure method, one can only take full advantage of the method accuracy in frequency domain analysis which is only suitable for linear elastic problems. This is mainly because the soil impedance functions are nonlinear functions of frequency and, in time domain analyses, they need to be convolved with the so-called foundation input motion (FIM) to map the correct traction along the interface between structure foundation and soil.

To take advantage of the substructure method in time domain analyses, researchers and practitioners have proposed different models to approximate the soil impedance functions (Stewart et al., 1999a, 1999b, 2012). For building structures on shallow foundations as shown in Figure 1a, the National Institute of Standards and Technology (NIST) under the project entitled Improved Procedures for Characterizing and Modeling Soil-Structure Interaction for Performance-Based Seismic Engineering recommended using single-valued functions at a representative frequency, which can be modeled as constant-valued springs and dashpots distributed along the soil-structure interface (see Figure 1b). This representative frequency is computed using an iterative method proposed by Bielak (1974); Veletsos and Nair (1975): first, as shown in Figure 1c, the fixed-base building period (i.e. T) and building structure geometry are used to compute the dimensionless frequency $a_{0}=\omega B / V_{s}$, where $\omega$ is the angular frequency of the interaction, $B$ is the half-width, and $V_{s}$ is the shearwave velocity. The lumped translational and rotational spring and dashpot coefficients are 


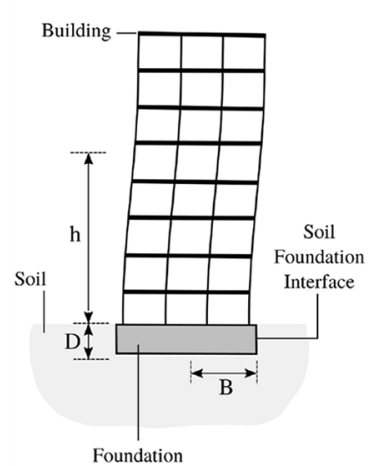

(a)

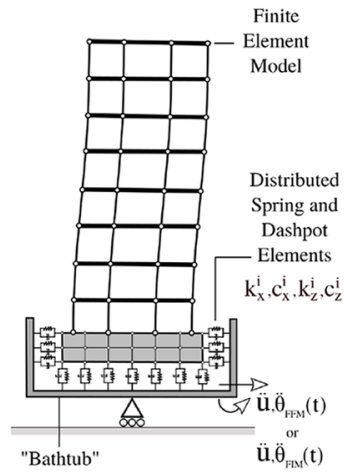

(b)

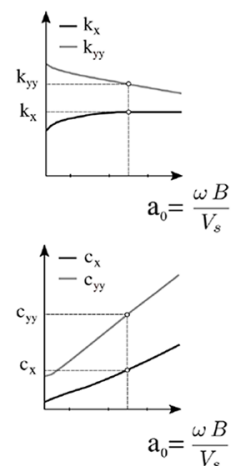

(c)

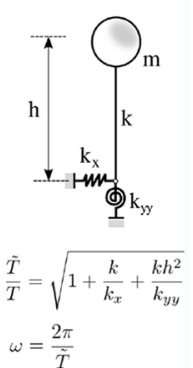

(d)

Figure I. NIST procedure to compute frequency-independent foundation soil spring and dashpot coefficients. (a) The real SSI problem. (b) Distributed soil spring $k_{x}^{i}$ (horizontal), $k_{z}^{i}$ (vertical) and dashpot $c_{x}^{i}$ (horizontal), and $c_{z}^{i}$ (vertical) along the foundation perimeter. (c) Real (top) and imaginary (bottom) components of the frequency-dependent impedance function. (d) Simplified SSI model using lumped translational and rotational spring and dashpots and the fixed first modal information.

next evaluated at this frequency, as shown in Figure 1d. Next, the lumped soil impedance coefficients are used to compute the period elongation of the system as follows:

$$
\frac{\tilde{T}}{T}=\sqrt{1+\frac{k}{k_{x}}+\frac{k h^{2}}{k_{y y}}},
$$

where the variable $\tilde{T}$ is the flexible-base period, $T$ is the fixed-base period, $h$ is the firstmodal height, $k$ is the fixed building first modal stiffness, and $k_{x}$ and $k_{y y}$ are the lumped translational and rotational spring coefficients that account for the flexibility of the surrounding soil. The process is repeated until the change in period elongation estimated in two consecutive iterations is below a certain threshold. Finally, the foundation impedance function-the lumped soil springs $k_{x}, k_{y y}$ and dashpots $c_{x}, c_{y y}$-are evaluated at the converged flexible-base period and if necessary, are distributed along the foundation perimeter as shown schematically in Figure 1b. The period elongation equation adopted in NIST has been introduced by Veletsos and Meek (1974), Bielak (1974), and Wolf (1985) and unfortunately does not include the coupled rotational-translational foundation stiffness $k_{x y}$. This term becomes important for deep embedded foundations as presented by Gazetas (1996). Therefore, other expressions (e.g. Maravas et al., 2014; Zania, 2014) can be used in a similar manner to improve NIST accuracy.

To modify the FFM due to vertically propagating shear waves that take the foundation embedment effects into account, NIST recommends using the zero-phase transfer functions $\mathcal{H}_{u}$ and $\mathcal{H}_{y y}$ originally proposed by Kausel et al. (1978) and Day (1978), which reduce the translational and introduce rotational motion to the FIM, respectively.

$$
\mathcal{H}_{u}=\left\{\begin{array}{l}
\cos \left(\frac{D \omega}{V_{s}}\right), \text { if } \frac{D \omega}{V_{s}}<1.1 \\
0.45, \text { otherwise }
\end{array}\right.
$$




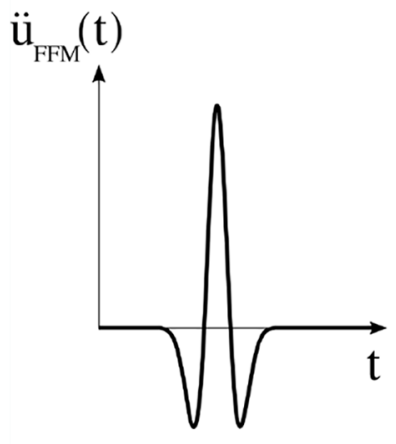

(a)

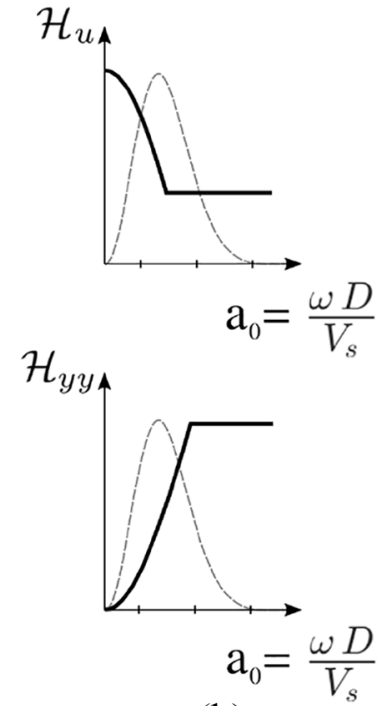

(b)
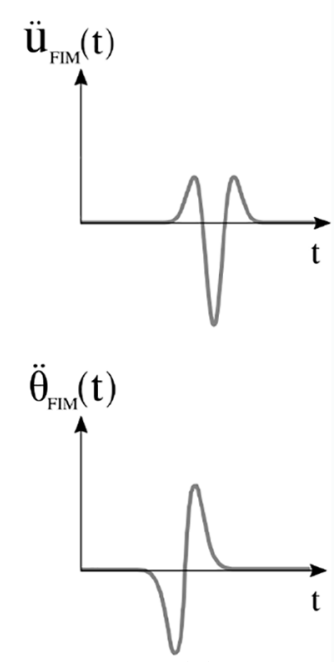

(c)

Figure 2. NIST procedure to compute foundation input motion. (a) Free field motion time series, here a Ricker pulse is considered. (b) Transfer function in black-solid line and FFM Fourier transform function of the Ricker pulse in dashed-gray line. (c) Generated translational and rotational foundation input motion.

$$
\mathcal{H}_{y y}=\left\{\begin{array}{l}
0.26\left[1-\cos \left(\frac{D \omega}{V_{s}}\right)\right], \text { if } \frac{D \omega}{V_{s}}<\frac{\pi}{2}, \\
0.26, \text { otherwise }
\end{array}\right.
$$

In Equations 2 and 3, the variable $D$ represents the foundation embedment, $V_{s}$ the shear wave velocity, and $\omega$ the frequency. Figure 2 shows schematically the FFM to FIM mapping, where the FFM signal in Figure 2a is first transformed in the Fourier space (dashed gray line), then the Fourier coefficients are scaled by the translational and rotational transfer functions as shown in Figure $2 \mathrm{~b}$ (in solid black line), and the results are then transformed back to the real space (using the inverse Fourier transform) to obtain the FIM as shown in Figure 2c. Finally, the resulting FIM is applied to a rigid diaphragm (a.k.a bathtub) as shown in Figure 1b.

It is evident that the NIST recommendations make two important simplifications to enable the substructure method to be used in time domain analyses: (a) the impedance value is evaluated at the flexible-base frequency and (b) the FIM preserves the phase of the FFM, independent of the foundation embedment ratio $D / B$. Upon these simplifications, the following questions may arise:

1. How do time-domain analyses using the NIST procedure compare to the direct SSI method?

2. What is the optimal impedance and corresponding dimensionless frequency $a_{0}$ for implementation in time-domain analyses? 

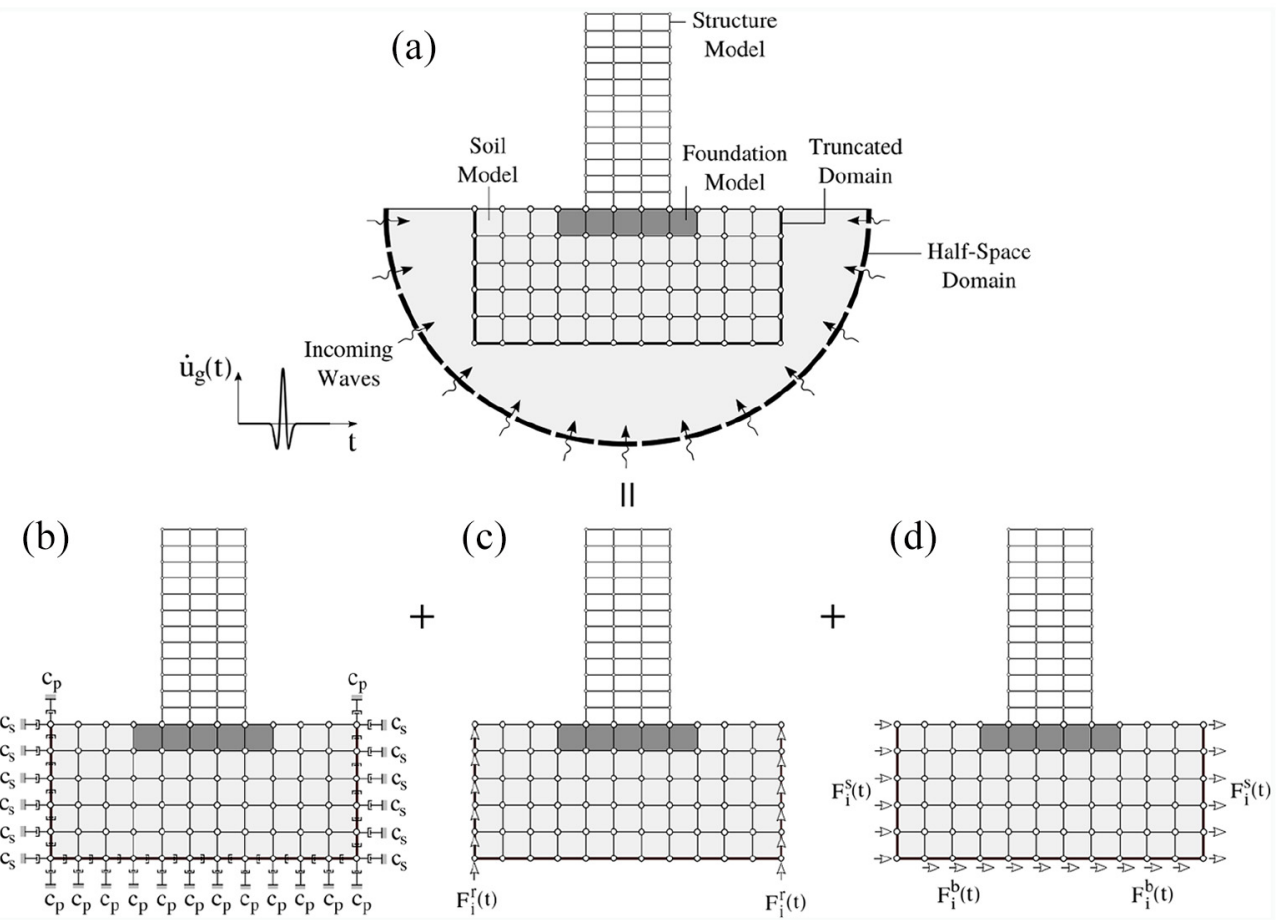

Figure 3. Reduced infinite half-space model using absorbing dashpots elements. (a) Semi-infinite soilstructure interaction problem, the solid-red line represents the truncated domain. (b) horizontal and vertical dashpots elements placed along the truncated domain. (c) reactive forces applied on the left and right boundary. (d) equivalent horizontal forces required to propagate the upward SV-wave.

The main objective of this study is to address the above listed questions. In the rest of this manuscript, we provide details of the approach we used to assess the performance of NIST recommendations for time domain modeling of SSI effects for two-dimensional (2D) building structures embedded in elastic half-space. We explain in detail the ground true (reference) solution; the derivation of a reduced order model (ROM) for inverse analyses; and the search of the parameter space of the ROM to the optimal impedance function values and the corresponding frequency. For the former, we model the problem using the direct method and for the latter we use a Bayesian approach based on the EnKI. Then, we use the developed framework to comprehensively assess the predictive capability of the NIST recommendations for a series of building structures. Finally, we summarize the findings of this study.

\section{Direct modeling of the problem}

For direct modeling of the 2D building structure embedded in elastic half-space, we use the procedure described in Figure 3. In the following sections, we first describe the building and soil finite element model (FEM), and then the far-field boundary conditions.

\section{FEM}

Three different topologies of buildings are considered, which are illustrated from the shortest in Figure 4a to the tallest in Figure 4c. The first reinforced concrete building has columns of rectangular cross section $0.90 \times 0.60 \mathrm{~m}$, each of $3.5 \mathrm{~m}$ height. Beams have 


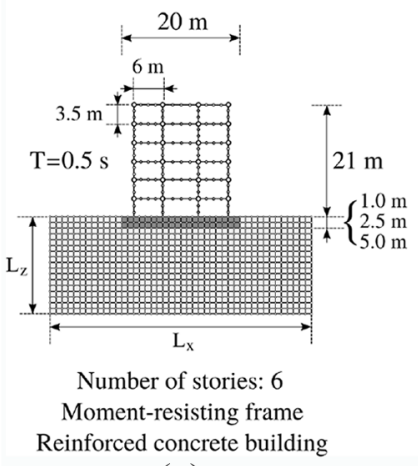

(a)

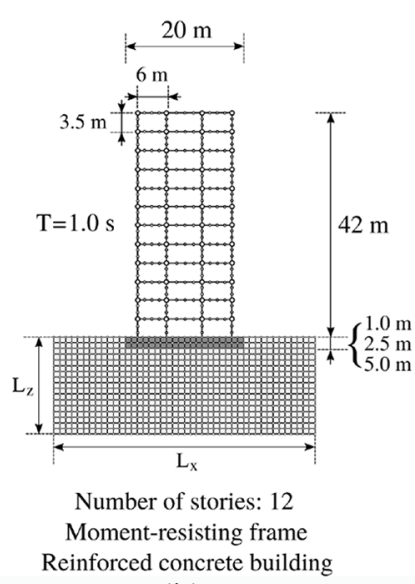

(b)

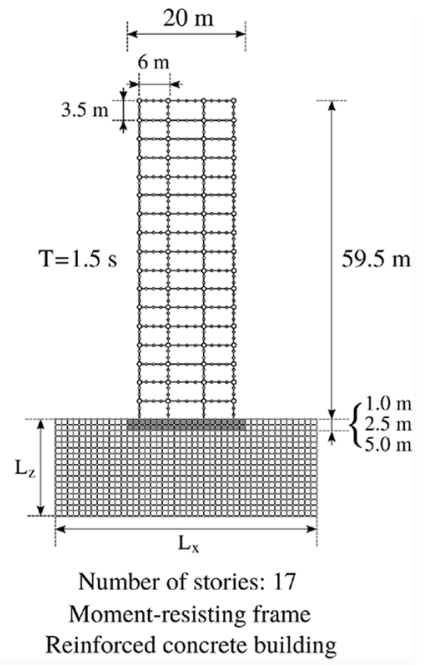

(c)

Figure 4. Building configurations employed to evaluate the true responses for the SSI problem. (a) Fixed-base period 0.5s. (b) fixed-base period I.0s. (c) fixed-base period I.5s

Table I. First fixed-modal-parameters using an eigen-analysis for three buildings

\begin{tabular}{llllll}
\hline \multicolumn{2}{l}{ Building parameters } & & & \multicolumn{2}{l}{ Eigen-analysis } \\
\cline { 1 - 2 } \cline { 5 - 6 } Period $(\mathrm{s})$ & Mass $(\mathrm{kg})$ & Height $(\mathrm{m})$ & & Modal mass $(\mathrm{kg})$ & Modal height $(\mathrm{m})$ \\
\hline 0.50 & $0.645 \cdot 10^{6}$ & 21.0 & & $0.545 \cdot 10^{6}$ & 15.0 \\
1.00 & $1.400 \cdot 10^{6}$ & 42.0 & & $1.150 \cdot 10^{6}$ & 30.0 \\
1.50 & $1.980 \cdot 10^{6}$ & 59.5 & & $1.480 \cdot 10^{6}$ & 40.0 \\
\hline
\end{tabular}

rectangular cross sections $0.80 \times 0.60 \mathrm{~m}$ and $6.0 \mathrm{~m}$ length. The reinforced concrete density is $2500 \mathrm{~kg} / \mathrm{m}^{3}$, the reinforced concrete elasticity modulus is taken as $25 \mathrm{GPa}$, and beams are subjected to a dead load of $3600 \mathrm{~kg} / \mathrm{m}$. This configuration gives a total height of $21 \mathrm{~m}$, a total mass of $6.45 \cdot 10^{5} \mathrm{~kg}$, a fixed-base fundamental period of $0.507 \mathrm{~s}$, a firstmodal height of $14.91 \mathrm{~m}$, and a first modal mass of $0.545 \cdot 10^{6} \mathrm{~kg}$. The solid core foundation has a half-width of $10 \mathrm{~m}$, an equivalent out-of-plane thickness of $1.0 \mathrm{~m}$, and three different embedment depths of $1.0,2.5$, and $5.0 \mathrm{~m}$ are considered, respectively. The reinforced concrete material properties for the foundation are taken such that the density provides a foundation mass $50.0 \cdot 10^{3} \mathrm{~kg}$, elasticity modulus $30 \mathrm{GPa}$, and Poisson's ratio of 0.25 . In this regard, a thick rigid mat foundation is considered and the concrete density is modified so that it emulates the basement levels. The foundation rigid diaphragm behavior is enforced by adding an auxiliary node with three degrees of freedom (i.e. diaphragm node) and then impose kinematic constraints to all nodes at the foundation and columns as suggested by Bathe (2006) and Cook et al. (2007). The second and third reinforced concrete buildings only differ in terms of the column cross-sections, which for the taller buildings are $1.00 \times 0.80 \mathrm{~m}$. Beams and material properties of building and foundation are same as above. The foundation is assumed to be a rigid diaphragm and to have perfect bonding with the surrounding soil. Table 1 summarizes some of the buildings' characteristics. 
The soil is represented as an elastic, homogeneous, and semi-infinite medium in plane strain setting with density $\rho_{s}=2000 \mathrm{~kg} / \mathrm{m}^{3}$, Poisson's ratio $\nu_{s}=0.25$, and shear wave velocity $V_{s}=80,100,125,150,175,200,225,250,300,400,500 \mathrm{~m} / \mathrm{s}$. The time step that satisfies stability constraints is $\Delta t=0.001 \mathrm{~s}$, and the simulation time is set to be $t_{\text {sim }}=30 \mathrm{~s}$. The spatial discretization is then taken as $\Delta x=\Delta z=0.5 \mathrm{~m}$, and the soil domain is truncated so that $L_{x}=350 \mathrm{~m}$ and $L_{z}=200 \mathrm{~m}$. The resulting finite element mesh has approximately 280,000 quadrilateral elements; we employed parallel computing for the simulations using an inhouse finite element code Seismo-VLab (Kusanovic et al., 2020); we divided the domain into seven sub-domains using the computer software OpenMPI ${ }^{1}$ (Broquedis et al., 2010; Gabriel et al., 2004; Hursey et al., 2007). The average time per simulation was 65 min approximately.

\section{Far-field boundary conditions}

We truncate the semi-infinite half-space as shown in Figure 3a and introduce Lysmer dashpots accompanied with prescribed nodal forces to artificially approximate an interface transparent to both incoming and outgoing waves (Assimaki, 2004; Lysmer et al., 1969). The Lysmer dashpot coefficient shown in Figure $3 \mathrm{~b}$ for absorbing outgoing waves is computed as:

$$
\begin{aligned}
& c_{s}=\rho_{s} V_{s} t_{h} \Delta l_{i}, \\
& c_{p}=\rho_{s} V_{p} t_{h} \Delta l_{i} .
\end{aligned}
$$

Similarly, the introduced nodal forces, shown in Figure $3 \mathrm{c}$ and $\mathrm{d}$, for translation of the vertically propagating incoming shear waves within the domain of interest are

$$
\begin{gathered}
F_{i}^{b}=2 c_{s} \dot{u}_{g}(t), \\
F_{i}^{s}=c_{p} \dot{u}_{i}^{s}(t), \\
F_{i}^{r}= \pm c_{s} \dot{u}_{i}^{r}(t) .
\end{gathered}
$$

In Equations 4 and 5, the variable $t_{h}=1.0 \mathrm{~m}$ is the out-of-plane thickness of the truncated soil domain, $\Delta l_{i}$ is either $\Delta x_{i}$ or $\Delta z_{i}$ and represent the tributary $i$ th side length of the element which are different for corner and inner boundary nodes, $V_{s}$ is the shear wave velocity of the soil, and $V_{p}$ is the compressive wave velocity in the soil.

It is worth pointing out that the boundary conditions enforced in this manner can almost perfectly absorb body waves with angles of incidence greater than $30^{\circ}$ with respect to the vertical axis, but lose their effectiveness for lower angles of incidence, including surface waves. Moreover, in Equations 6 to 8, the signals $\dot{u}_{g}(t)$ and $\dot{u}_{i}^{s}(t)$ represent the incident and the total free field motion for which a Ricker-wavelet is prescribed at the bottom of the model and given in Equation 9 as:

$$
\dot{u}_{g}(t)=\left(1-2 \beta\left(t-t_{0}\right)^{2}\right) \exp \left[-\beta\left(t-t_{0}\right)^{2}\right]
$$

where $\beta=\left(\pi f_{0}\right)^{2}, f_{0}$ is the characteristic frequency, and $t_{0}$ is the time position where the velocity will become maximum. In all simulations, we consider a characteristic frequency $f_{0}=2.0 \mathrm{~Hz}$ and a peak time velocity $t_{0}=1.0 \mathrm{~s}$. The characteristic frequency $f_{0}$ is selected to 

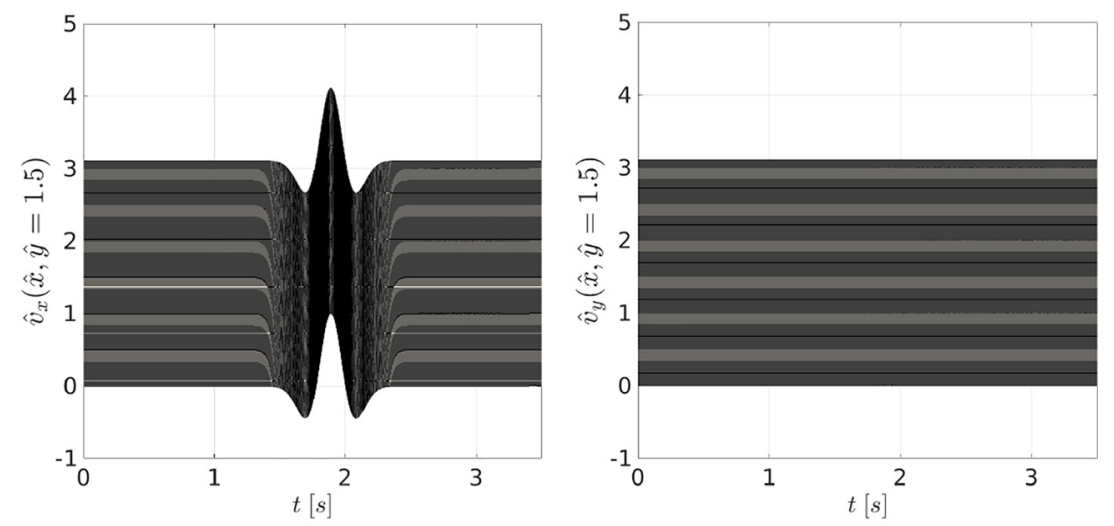

Figure 5. Synthetic seismogram for the soil-wave propagation problem (Left) normalized horizontal component $\hat{v}_{x}(t)$ and (Right) normalized vertical component $\hat{v}_{y}(t)$ of the velocity recorded on the soil surface.

generate an FFM with frequency content that ranges between $0-7.5 \mathrm{~Hz}$ and emulates the majority of real earthquake signals. Base slab averaging effects are not included in the simulations since the FFM consists of vertically propagating waves. To compute the horizontal and vertical forces on the left and right boundaries, the nodal velocities $\dot{u}_{i}^{s}(t)$ and $\dot{u}_{i}^{r}(t)$ are calculated as follows:

$$
\dot{u}_{i}^{s}(t)=\dot{u}_{g}\left(-\frac{z_{i}}{V_{s}}+t\right)+\dot{u}_{g}\left(\frac{z_{i}-2 H}{V_{s}}+t\right)
$$

and,

$$
\dot{u}_{i}^{r}(t)=\dot{u}_{g}\left(\frac{z_{i}-2 H}{V_{s}}+t\right)-\dot{u}_{g}\left(-\frac{z_{i}}{V_{s}}+t\right)
$$

where $z_{i}$ is the vertical coordinate of the $i$ th node measured with respect to the bottom of the soil domain, $H$ is the total vertical height of the truncated soil domain, and \pm sign in Equation 8 represents the left $(+)$ or right $(-)$ vertical boundary forces, respectively.

We verify our implementation by reproducing the free-field motion in a homogeneous half-space with length $L_{x}=350 \mathrm{~m}$ and height $L_{z}=200 \mathrm{~m}$. We consider linear elastic soil elements with $\rho_{s}=2000 \mathrm{~kg} / \mathrm{m}^{3}, \nu_{s}=0.25, t_{h}=1.0 \mathrm{~m}$, for which plane-strain conditions are enforced. The shear wave velocity is set to $V_{s}=225 \mathrm{~m} / \mathrm{s}$, leading to a wave-length of $\lambda_{n}=V_{s} / f_{0}=113 \mathrm{~m}$ corresponding to the dominant frequency of the wavelet. The results of the normalized velocity field $\hat{v}(\hat{x}, \hat{y})=v(\hat{x}, \hat{y}) / \max |v|$ as a function of the normalized coordinates $\hat{x}=x / \lambda_{n}, \hat{y}=y / \lambda_{n}$ are displayed in Figure 5 for both components of the velocity field. This figure makes evident that the implemented boundary interface works perfectly for this case. Here, one can observe that there are no reflections coming from the boundaries, and as expected, velocities in the vertical direction do not manifest. 


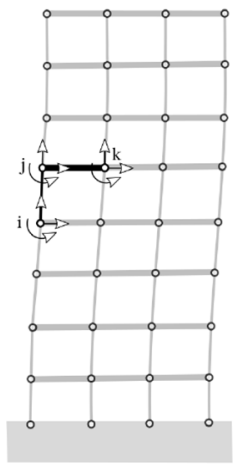

(a)

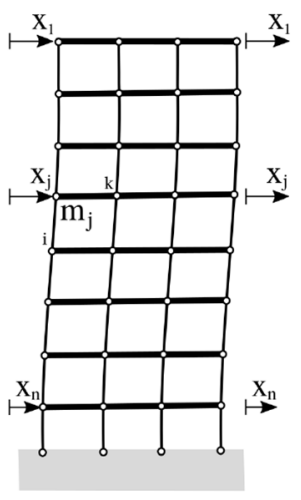

(b)

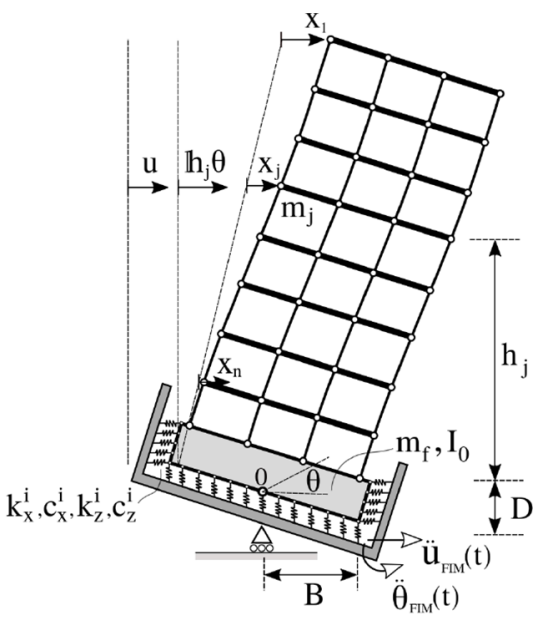

(c)

Figure 6. The reduced order modeling. (a) The column and beam elements used to construct the fixed base FEM. (b) The fixed-base FEM building model where $\mathbf{M}_{s}, \mathbf{C}_{s}, \mathbf{K}_{s}$ are obtained using static condensation. (c) The flexible-base soil-structure interaction system in which $x_{i}$ represents the relative displacement of each floor, $u$ represents the relative deformation of the soil, and $\theta$ the rigid rotation of the foundation.

\section{Reduced order modeling of the problem}

For the substructure method, we consider the 2D ROM shown in Figure 6c.

The ROM is constructed to preserve the modal characteristics of the fixed base building (Asimaki et al., 2019; Kusanovic et al., 2018). To this end, we employ frame elements with three degrees of freedom per node to represent the structure's geometry as shown in Figure 6a. We assume that each floor acts as a rigid diaphragm, so that the buildings mass can be lumped at the floor levels as shown in Figure 6b. We then use static condensation (Guyan, 1965; Irons, 1965) on the fixed-base building to compute the $\mathbf{M}_{s}, \mathbf{C}_{s}, \mathbf{K}_{s} \in \mathbb{R}^{n \times n}$ matrices where $n$ is the number of floors; therefore, the vector $x \in \mathbb{R}^{n}$ represents the horizontal degree of freedom of the building, since there is only one translational degree of freedom on each floor. In addition, we assume a rigid rectangular foundation of half-width $B$ and embedment depth $D$ on an elastic half-space, for which two additional degrees of freedom $u, \theta \in \mathbb{R}$ represent the translation and rotation of the foundation resulting from the soil compliance. The foundation has a total mass $m_{f}$ and a moment of inertia $I_{0}$. Then, depending on the excitation, we consider two variants for forward modeling: (1) excited by FIM and (2) excited by FFM. The semi-discrete equations of motion for both cases are:

$$
\begin{aligned}
& {\left[\begin{array}{ccc}
\mathbf{M}_{s} & \mathbf{M}_{s} \mathbb{1} & \mathbf{M}_{s} \rrbracket^{\mathrm{h}} \\
\mathbb{1}^{\mathrm{T}} \mathbf{M}_{s} & m_{f}+\mathbb{1}^{\mathrm{T}} \mathbf{M}_{s} \mathbb{1} & m_{f} \frac{D}{2}+\mathbb{h}^{\mathrm{T}} \mathbf{M}_{s} \mathbb{1} \\
\mathbb{n}^{\mathrm{T}} \mathbf{M}_{s} & m_{f} \frac{D}{2}+\mathbb{h}^{\mathrm{T}} \mathbf{M}_{s} \mathbb{1} & I_{0}+m_{f} \frac{D^{2}}{4}+\mathbb{h}^{\mathrm{T}} \mathbf{M}_{s} \mathbb{n}^{0}
\end{array}\right]\left[\begin{array}{c}
\ddot{x} \\
\ddot{v} \\
\ddot{\theta}
\end{array}\right]+} \\
& {\left[\begin{array}{ccc}
\mathbf{C}_{s} & \mathbb{O}_{n \times 1} & \mathbb{O}_{n \times 1} \\
\mathbb{O}_{1 \times n} & c_{x} & c_{x y} \\
\mathbb{O}_{1 \times n} & c_{x y} & c_{y y}
\end{array}\right]\left[\begin{array}{c}
\dot{x} \\
\dot{v} \\
\dot{\theta}
\end{array}\right]+\left[\begin{array}{ccc}
\mathbf{K}_{s} & \mathbb{O}_{n \times 1} & \mathbb{O}_{n \times 1} \\
\mathbb{O}_{1 \times n} & k_{x} & k_{x y} \\
\mathbb{O}_{1 \times n} & k_{x y} & k_{y y}
\end{array}\right]\left[\begin{array}{c}
x \\
v \\
\theta
\end{array}\right]=\mathbf{F}(t),}
\end{aligned}
$$


where the external force vector for the free field motion is

$$
\mathbf{F}(t)=-\left[\begin{array}{c}
\mathbf{M}_{s} \mathbb{1} \\
m_{f}+\mathbb{1}^{\mathrm{T}} \mathbf{M}_{s} \mathbb{1} \\
m_{f} \frac{D}{2}+\mathbb{h}^{\mathrm{T}} \mathbf{M}_{s} \mathbb{1}
\end{array}\right] \ddot{u}_{F F M(t)}
$$

and for the foundation input motion is

$$
\mathbf{F}(t)=-\left[\begin{array}{c}
\mathbf{M}_{s} \mathbb{1} \\
m_{f}+\mathbb{1}^{\mathrm{T}} \mathbf{M}_{s} \mathbb{1} \\
m_{f} \frac{D}{2}+\mathbb{h}^{\mathrm{T}} \mathbf{M}_{s} \mathbb{1}
\end{array}\right] \ddot{u}_{F I M(t)}-\left[\begin{array}{c}
\mathbf{M}_{s} \mathbb{b} \\
m_{f} \frac{D}{2}+\mathbb{1}^{\mathrm{T}} \mathbf{M}_{s} \mathbb{b} \\
m_{f} \frac{D^{2}}{4}+\mathbb{b}^{\mathrm{T}} \mathbf{M}_{s} \mathbb{b}_{\mathrm{h}}+I_{0}
\end{array}\right] \ddot{\boldsymbol{\theta}}_{F I M(t)},
$$

and $\ddot{x}, \dot{x}, x \in \mathbb{R}^{n}$ are the acceleration, velocity, and displacement vector for the condensed horizontal degrees of freedom of the building, also $\ddot{v}, \dot{v}, v \in \mathbb{R}$ and $\ddot{\theta}, \dot{\theta}, \theta \in \mathbb{R}$ are the foundation horizontal and rotational acceleration, velocity, and displacement. Note that we define $\mathbb{1} \in \mathbb{R}^{n}$ the vector of ones, this is $\mathbb{1}=(1,1, \ldots, 1)$, and $\mathbb{b} \in \mathbb{R}^{n}$ the vector of height, this is $\mathbb{W}_{0}=\left(h_{1}+D, \ldots, h_{n}+D\right)$. We finally define $\mathbb{O}_{n \times m} \in \mathbb{R}^{n \times m}$ the matrix of zeros.

We should highlight here that in the ROM, the horizontal $k_{x}^{i}$ and vertical $k_{z}^{i}$ springs and dashpots elements are distributed uniformly over the foundation perimeter. However, the contribution can be lumped as follows for both stiffness and damping components:

$$
\begin{aligned}
& k_{x}=2 k_{x}^{i}(D+B), k_{x y}=k_{x}^{i} D^{2}, k_{y y}=\frac{2}{3} k_{x}^{i} D^{3}+\frac{2}{3} k_{z}^{i} B^{3}+2 k_{z}^{i} B^{2} D, \\
& c_{x}=2 c_{x}^{i}(D+B), c_{x y}=c_{x}^{i} D^{2}, c_{y y}=\frac{2}{3} c_{x}^{i} D^{3}+\frac{2}{3} c_{z}^{i} B^{3}+2 c_{z}^{i} B^{2} D .
\end{aligned}
$$

Distributing the spring and dashpot elements as represented in Figure 6 allows us to take the coupling effects in the stiffness and damping matrices into account. We next describe how the stiffness and damping coefficients are computed using the NIST procedure and the methodology proposed in this article.

\section{NIST methodology}

The NIST methodology presented in Stewart et al. (2012) only provides expressions for impedance functions and FIM transfer functions in the three-dimensional (3D) setting. However, the same procedure can equally be applied to $2 \mathrm{D}$ plane-strain cases using the impedance functions and FIM transfer functions for the $2 \mathrm{D}$ case. To make a fair comparison, we first derived the "true" 2D impedance and FIM transfer functions in a planestrain setting using the methodology proposed by Seylabi (2016) and Seylabi et al. (2016). The numerically computed impedance functions and FIM transfer functions are presented in Figures 7 to 9, respectively. Please note that we only use $k_{x}$ and $k_{y y}$ components within the NIST framework and read them at the representative frequency considered for analysis. However, it should be noted that we use both the real and imaginary components (or equivalently the amplitude and phase angle) of $\mathcal{H}_{u}$ and $\mathcal{H}_{y y}$ to compute the FIM to be used in Equation 14. In Figure 7, we only represent the amplitude of the TFs, and we verify them against results presented in Conti et al. (2018). With these considerations, we here assess the NIST procedure's errors associated with reading impedance functions at a nonrepresentative frequency as well as those associated with ignoring the coupling terms. 

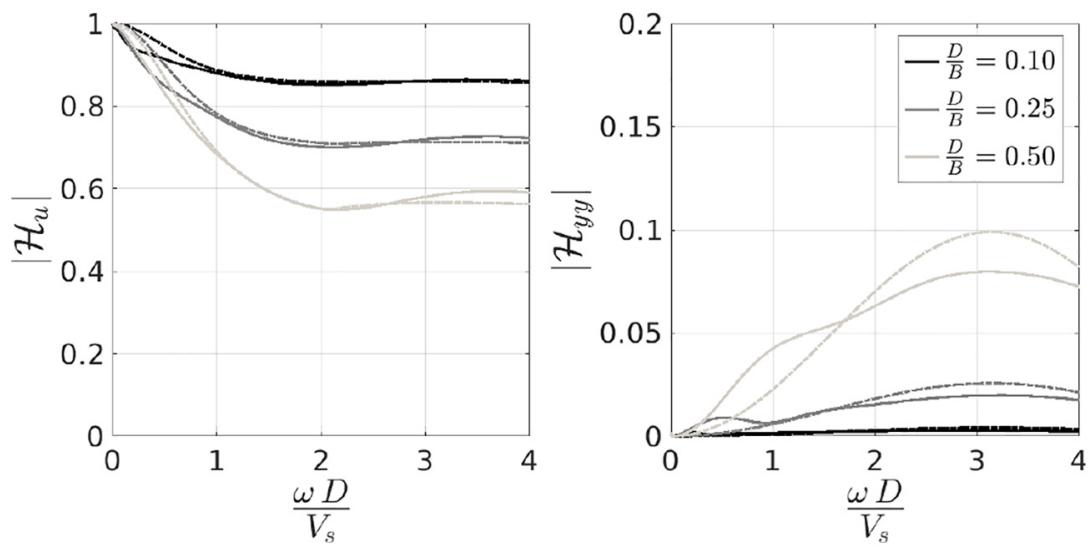

Figure 7. Foundation input motion (FIM) transfer functions (TFs) for different embedment ratio. $\mathcal{H}_{u}$ represents the translational FIM amplitude and $\mathcal{H}_{y y}$ represents the rotational FIM amplitude. The solid line represents the corresponding variable computed using Seylabi (20I6), while the dashed line are the corresponding variable presented in Conti et al. (2018).
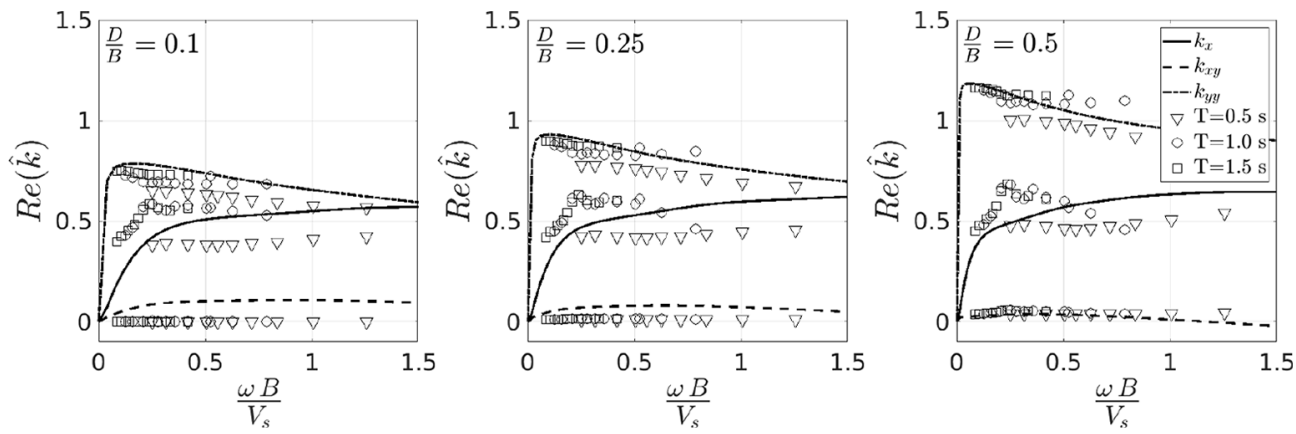

Figure 8. Identified lumped impedance (real part) computed for soil properties $\nu_{s}=0.25$ and $\rho_{s}=2000 \mathrm{~kg} / \mathrm{m}^{3}$ using EnKI (in discrete markers) and NIST (in solid line) for buildings with fixed base period $T=0.5,1.0,1.5 \mathrm{~s}$ and foundation embedment $D=1.0,2.5,5.0 \mathrm{~m}$.

\section{EnKI-based methodology}

For Equation 12 to provide accurate results, that is, closest responses to the direct modeling approach, the spring coefficients $k_{x}^{i}, k_{z}^{i}$ and dashpots coefficients $c_{x}^{i}, c_{z}^{i}$ need to be computed within an optimization framework. In order to find the optimal spring and dashpot coefficients of the ROM, we use the Bayesian approach based on the ensemble Kalman inversion (EnKI) (Evensen, 2006; Iglesias et al., 2013; Law et al., 2016), often referred to as particle-based derivative-free sequential optimization method. In the inversion setting, we consider the problem of finding $u \in \mathbb{R}^{n}$ from $y \in \mathbb{R}^{m}$ where

$$
y=\mathbf{G}(u)+\eta
$$

The variable $u \in \mathbb{R}^{n}$ consists of all the unknown parameters that we want to estimate, the variable $y \in \mathbb{R}^{m}$ consists of the ground truth quantities of interest, and $\eta$ is a zero-mean 

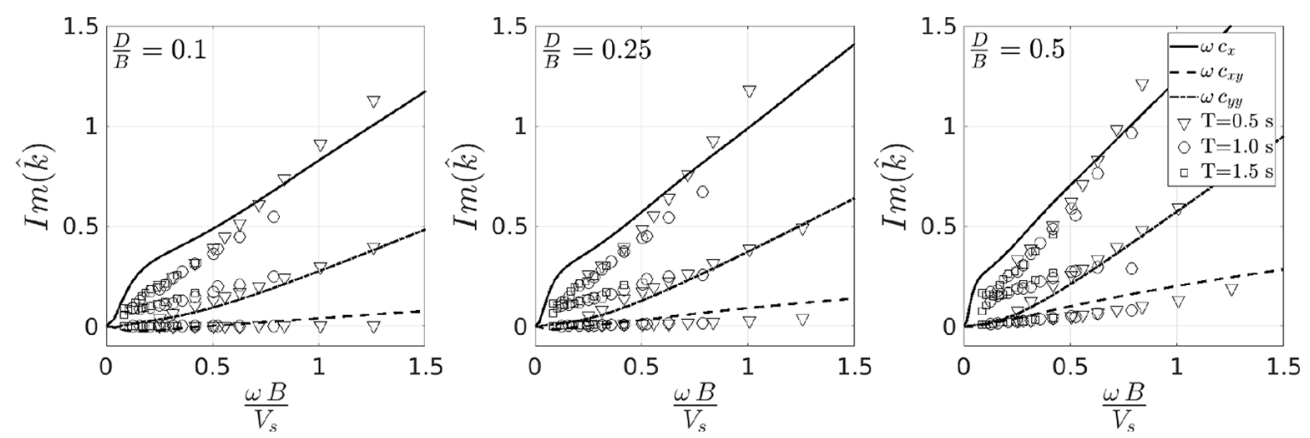

Figure 9. Identified lumped impedance (imaginary part) computed for soil properties $\nu_{s}=0.25$ and $\rho_{s}=2000 \mathrm{~kg} / \mathrm{m}^{3}$ using EnKI (in discrete markers) and NIST (solid line) for buildings with fixed base period $T=0.5,1.0,1.5 \mathrm{~s}$ and foundation embedment $D=1.0,2.5,5.0 \mathrm{~m}$.

Gaussian noise with covariance $\Gamma$. The nonlinear function (a.k.a. forward model) $\mathbf{G}: \mathbb{R}^{n} \rightarrow \mathbb{R}^{m}$ maps the parameter space to the data space. In this article, we work with one type of observation: the displacement time series recorded at the floors and foundation levels computed using the direct modeling method. Given $N$ particles $u_{j}^{(n)}, n=1, \ldots, N$ within the ensemble and at each iteration $j$, we use the predictions $\mathbf{G}\left(u_{j}^{(n)}\right)$ by the forward model, that is, the displacement time series computed by the ROM, and the observation data $y$ to update the particles for iteration $j+1$ (see in Appendix 1Figure 18 for schematic illustration). That is,

$$
u_{j+1}^{(n)}=u_{j}^{(n)}+\mathbf{C}_{j+1}^{u w}\left(\mathbf{C}_{j+1}^{w w}+\Gamma\right)^{-1}\left(y_{j+1}^{(n)}-\mathbf{G}\left(u_{j}^{(n)}\right)\right), \text { for } n=1, \ldots, N
$$

In Equation 18, $y_{j+1}^{(n)}$ can be either identical to $y$ (the observation data) or randomly perturbed using zero-mean Gaussian noise $\eta$; $\mathbf{C}_{j+1}^{u w}$ and $\mathbf{C}_{j+1}^{w w}$ are empirical covariance matrices that can be computed at each iteration based on predictions and the ensemble mean $\bar{u}_{j+1}$ using the following equations:

$$
\begin{gathered}
\mathbf{C}_{j+1}^{u w}=\frac{1}{N} \sum_{n=1}^{N}\left(u_{j}^{(n)}-\bar{u}_{j+1}\right) \otimes\left(\mathbf{G}\left(u_{j}^{(n)}\right)-\overline{\mathbf{G}}_{j}\right) \\
\mathbf{C}_{j+1}^{w w}=\frac{1}{N} \sum_{n=1}^{N}\left(\mathbf{G}\left(u_{j}^{(n)}\right)-\overline{\mathbf{G}}_{j}\right) \otimes\left(\mathbf{G}\left(u_{j}^{(n)}\right)-\overline{\mathbf{G}}_{j}\right)
\end{gathered}
$$

and

$$
\bar{u}_{j+1}=\frac{1}{N} \sum_{n=1}^{N} u_{j}^{(n)}, \quad \overline{\mathbf{G}}_{j}=\frac{1}{N} \sum_{n=1}^{N} \mathbf{G}\left(u_{j}^{n}\right)
$$

We should note here that the convergence criterion was defined as either reaching a relative change of $0.1 \%$ in all the parameters in two consecutive EnKI iterations, or a maximum number of 500 iterations. Note that the initial ensemble mean is set equal to the lumped stiffness and dashpot coefficients obtained using the NIST (Stewart et al., 2012) procedure. In addition, since the prior distribution of the soil parameters is unknown, we 
use a uniform distribution centered at the computed NIST value with lower and upper limits 10 times smaller and larger to represent this uncertainty. Finally, the positiveness of the stiffness and dashpot coefficients is enforced through a change of variables $u \in \mathbb{R}$, $\exp (u): \mathbb{R} \rightarrow[0, \infty]$ on which the EnKI is applied. The ROM constructed using EnKI and subjected to FFM or FIM are named hereafter as EnKI-FFM or EnKI-FIM, respectively.

\section{ROM performance assessment}

\section{Soil impedances of ROMs}

To estimate the constant valued soil impedances, the ROM is subjected to the FIM and the EnKI is employed to estimate the soil spring and dashpot coefficients. Then, they are compared against the true 2D impedance functions derived numerically using the method proposed by Seylabi et al. (2016). These EnKI-FIM results are displayed in Figures 8 and 9 in which the vertical axis has been normalized such that $\hat{k}_{x}=\pi G_{s}\left(k_{x}+\omega c_{x}{ }^{0}\right)$, $\hat{k}_{x y}=\pi G_{s} B\left(k_{x y}+\omega c_{x y} \mathrm{~d}\right)$, and $\hat{k}_{y y}=\pi G_{s} B^{2}\left(k_{y y}+\omega c_{y y} \mathrm{~d}\right)$, where $i^{2}=-1, B$ is the half-width foundation, $G_{s}=\rho_{s} V_{s}^{2}$ is the soil shear modulus, $\rho_{s}$ the soil's density, and $V_{s}$ the soil shear wave velocity.

We also employ the EnKI to re-estimate the soil springs and dashpots when the system is subject to FFM. Considering the EnKI-FIM results as the optimal solution for the considered ROM, we next assess the accuracy of the EnKI-FFM results as well as those obtained using NIST procedure. In Figure 10, the horizontal axis is the EnKI-FIM results while the vertical axes are the EnKI-FFM and NIST results. This comparison study delineated that

1. The embedment effect is almost negligible as the EnKI-FIM and EnKI-FFM results are highly correlated with each other, which in turn confirms that the embedment ratios considered in this study are shallow.

2. The NIST results capture very well the total lumped horizontal $k_{x}$ and rotational $k_{y y}$ spring coefficients. However, the total lumped horizontal $c_{x}$ and rotational $c_{y y}$ dashpot coefficients are not equally well estimated. The least well estimated are the lumped rotational dashpot coefficients for tallest building, with $T=1.5 \mathrm{~s}$. This problem arises because the imaginary component of the lumped rotational impedance $c_{y y}$ is inversely proportional to the fixed building period; consequently, for very low dimensionless frequencies, the imaginary component of the lumped rotational impedances is approximately one order of magnitude smaller compared to the EnKI-FIM results, as shown in Figure 10. This suggests that the frequency at which flexible buildings radiate energy due to rocking is different from the swaying mechanism proposed by NIST, which is associated with the flexible base response.

The previous analysis made clear that one can improve upon the frequency recommendation used to estimate the imaginary component of the soil impedances. We specifically showed that if the flexible-base period is used to compute the lumped rotational dashpot coefficient for flexible buildings, the radiation damping is underestimated. In the remainder of this section, we seek the optimal dimensionless frequency (based on the flexible-base period $\tilde{T}$, fixed-base period $T$, or the input signal dominant frequency $f_{0}$ ) that can be used to evaluate the foundation impedance functions. As before, we perform numerical analyses to answer this question, but now we consider only the NIST recommendation using these three frequencies. 

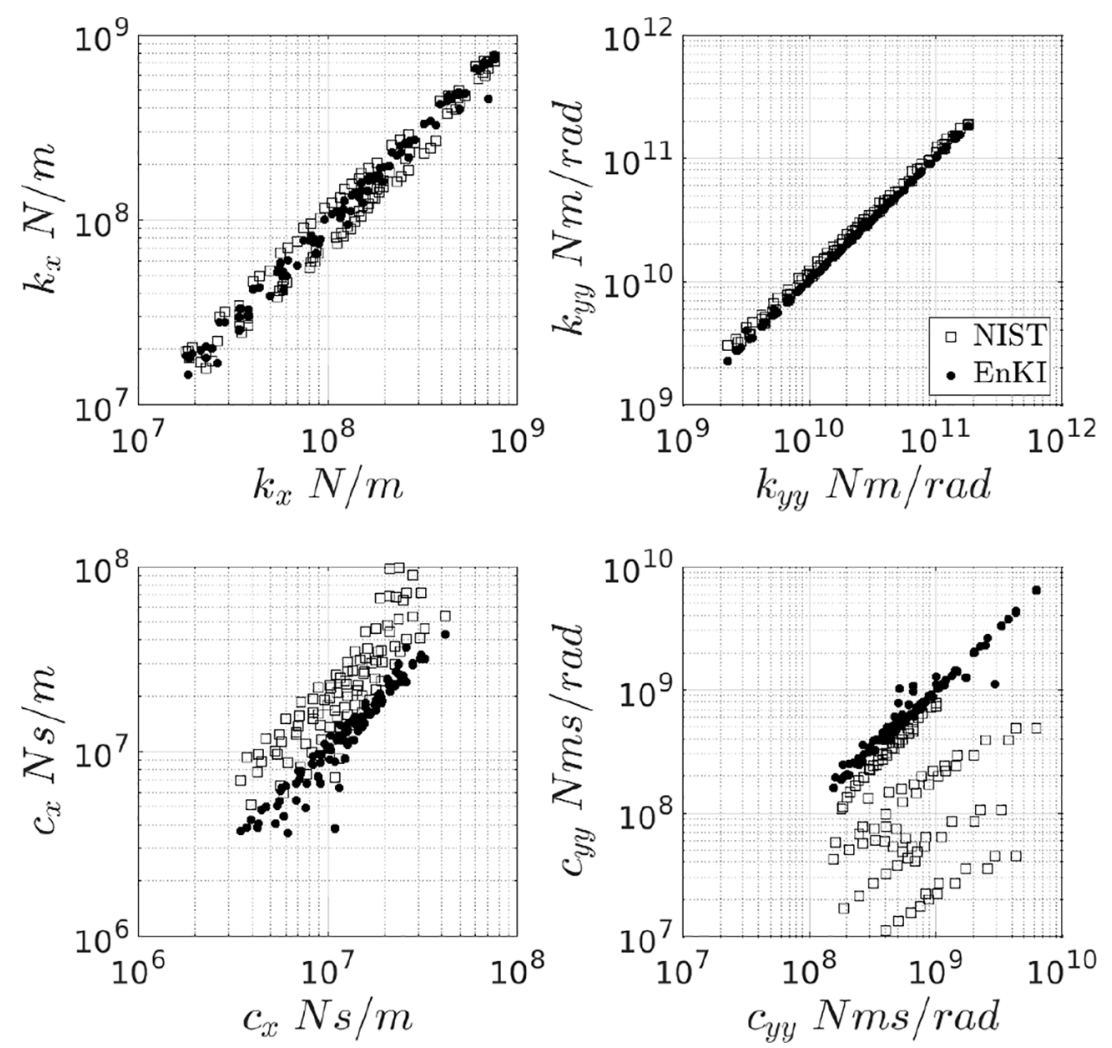

Figure 10. Identified impedance component comparison between NIST and EnKI using FIM and FFM. The horizontal axis correspond to the EnKI once the system is subject to FIM. The vertical axis represents the impedance components using NIST (in white squares) and EnKI subjected to FFM (in black solid dots).

In Figure 11, we compare the NIST-recommended soil spring and dashpot coefficients (vertical axis) against the EnKI-FIM results on the horizontal axis. Once again, we notice that the horizontal and rotational spring coefficients are estimated properly, no matter which frequency is used. However, a smaller variability is obtained if the fixed-base period is employed. However, the lumped horizontal and vertical dashpot coefficients show high sensitivity to the building and foundation geometry and stiffness. In general, the input signal dominant frequency $f_{0}$ as well as the fixed-base period $T$ perform better in estimating the lumped vertical dashpot coefficient. Here as well, the main difference in the time history response is associated with the lumped rotational dashpot coefficient that is poorly estimated. In order to show this point, we quantify the total deviation of the dynamic response of the full FEM model (as described in direct model section); and the two ROMs, one with the NIST-recommended spring and dashpot coefficients, and one with the EnKI-FIM impedances, for the three structures of interest. We measure the $\ell_{2}$-error for each case as:

$$
\|y-\hat{y}\|_{\ell_{2}}^{2}=\frac{1}{N_{t} N_{m}} \sum_{k=1}^{N_{m}} \sum_{j=1}^{N_{t}}\left(y_{j}^{(k)}-\hat{y}_{j}^{(k)}\right)^{2} \text {, }
$$



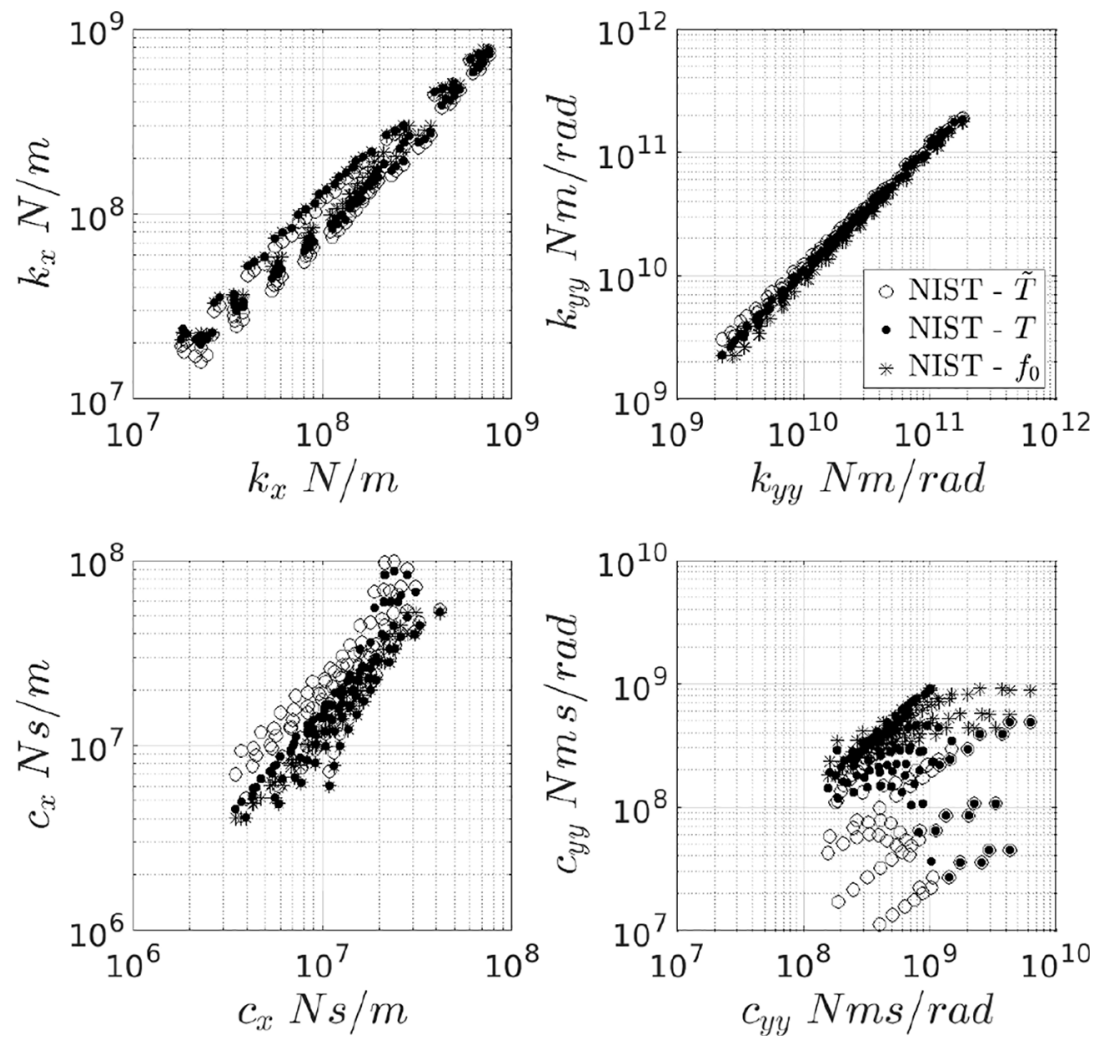

Figure I I. Impedance comparison between NIST using the flexible base period $\tilde{T}$ (in white circles), fixed base period $T$ (in black solid dots), or the input signal dominant frequency $f_{0}$ (in asterisk) and EnKI using foundation input motion.

where $y_{j}^{(k)}$ represents the $k$ th observation (from the FEM) at $j$ th time step, $\hat{y}_{j}^{(k)}$ represents the $k$-th response (from the ROM) at $j$ th time step, $N_{t}$ corresponds to the number of time steps, and $N_{m}$ represents the number of observations considered. We consider a time series of $15 \mathrm{~s}$ with a sampling time step $\Delta t=0.01 \mathrm{~s}$ for the translation and rotation of the foundation, and the horizontal translation of each floor. Figure 12 represents the total $\ell_{2}$-error in the vertical axis, and the shear wave velocity in the horizontal axis. Note that in overall the $\ell_{2}$-norm error in the vertical axis is small because the displacements are of the order of centimeters; however, a difference of almost two orders of magnitude is obtained for some cases between the NIST and EnKI. This figure also shows that, in general, the NIST model using the fixed-base frequency and the signal predominant frequency yields smaller errors relative to the flexible-base case. The latter is smaller only for the building with period $T=1.0 \mathrm{~s}$ and embedment $D=5.0 \mathrm{~m}$. However, none of the configurations reached the error obtained using EnKI-FIM. The discrepancies are attributed mostly to the poor estimation of the lumped rotational dashpot coefficients, which generate more oscillations in the transient part, and take longer to be damped out in the free oscillation part. These discrepancies are evident in Figure 13, where the NIST building responses are compared against the direct modeling approach. Note how the estimated NIST frequency (a) underestimates the inter-story drift ratio and the shear force at each floor, and (b) fails to capture the global period elongation and radiation damping for the displacement response. 


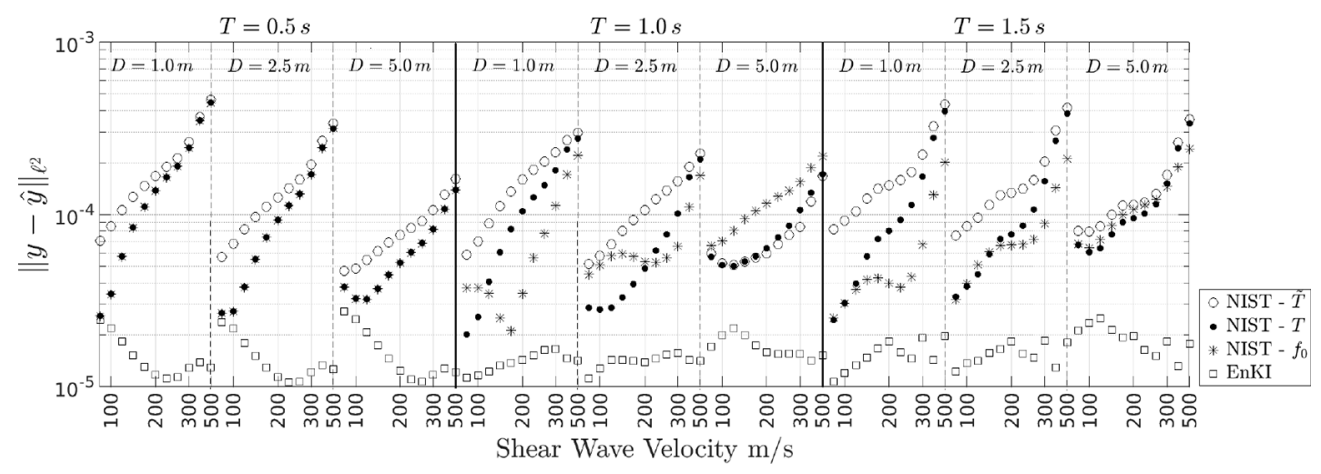

Figure 12. Total time history error measured in $\ell_{2}$-norm for the three practical dimensionless frequencies suggested in NIST. In white circle, the flexible base period, in solid black-dots the fixed-base period, in asterisk the signal-predominant frequency, and in white square the ensemble Kalman inversion.
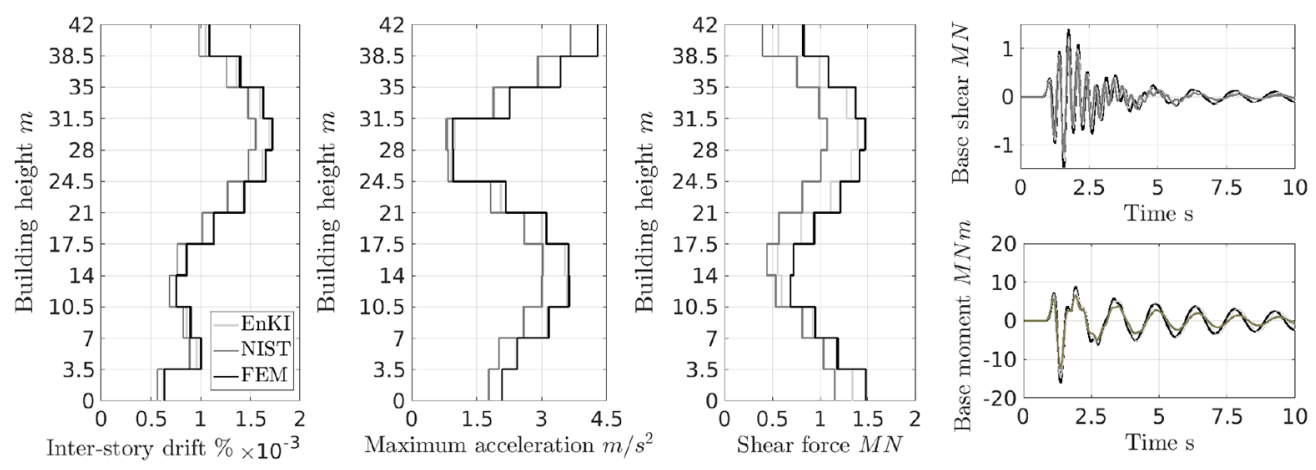

Figure 13. Comparison between direct modeling (FEM), EnKI, and NIST for different building responses. The building has a fixed-base period of $T=1.0 \mathrm{~s}$ and is shown in Figure 4b. The foundation's dimension are $B=10 \mathrm{~m}$ and $D=1 \mathrm{~m}$. The soil properties used in the analysis are $V_{s}=300 \mathrm{~m} / \mathrm{s}$ and $\nu_{s}=0.25$.

\section{Dynamic global properties}

Using the NIST and results of soil impedance obtained using EnKI-FIM, we computed the period elongation ratio $\tilde{T} / T$ and foundation-damping $\beta_{f}$ that the ROM experiences when supported on lumped spring and dashpot elements (compared to fixed base conditions). Since the proposed ROM considers approximately the coupling between the translational and rotational degrees of freedoms, we employ Equations 31 and 37 derived in Appendix 1 to estimate the period lengthening and foundation damping. We then compare these results against the iterative method summarized in the "Introduction" section.

Figures 14 and 15 show the period elongation and foundation damping computed using the two methods. Results show a good-agreement between the proposed expressions in the Appendix 1 and the NIST-proposed expressions, derived using the iterative method. From these figures, we note that Equation 31 results in a more flexible system compared to Equation 37, which yields a less-dissipative system when they are compared with the NIST recommendations. A very close fit is, however, obtained for the three buildings when the foundation aspect ratio is small (i.e. $D / B=0.10$ ). The discrepancies must be attributed to the following facts: 

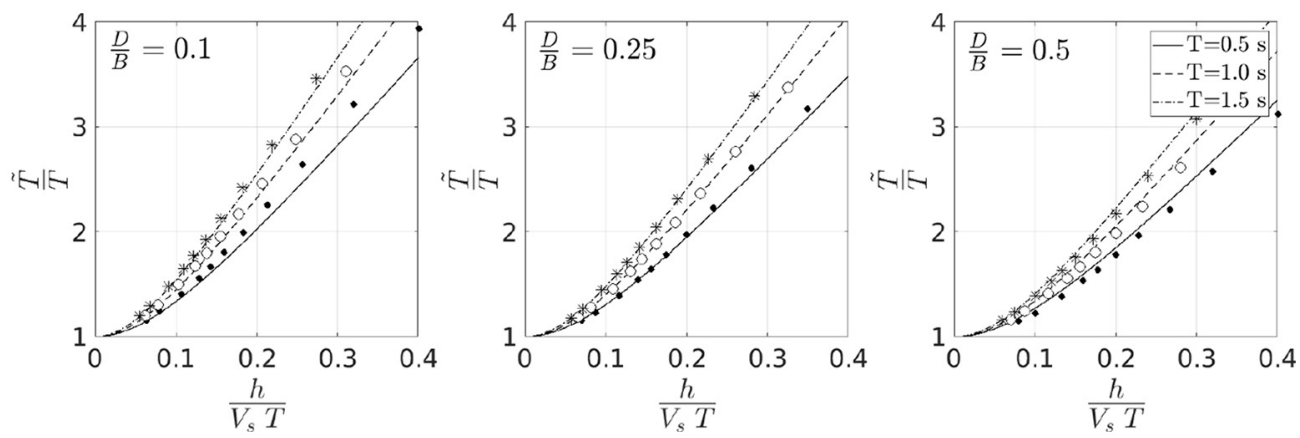

Figure 14. Identified period elongation ratio $\hat{T} / T$ computed for soil properties $\rho_{s}=2000 \mathrm{~kg} / \mathrm{m}^{3}$ and $\nu_{s}=0.25$ using EnKI (markers) and NIST (lines) for building of fixed-base period $T=0.5,1.0,1.5 \mathrm{~s}$, first modal height $h=15,30,45 \mathrm{~m}$, foundation embedment $D=1.0,2.5,5.0 \mathrm{~m}$, and supported on eleven soils with shear velocity $V_{s}=80,100,125,150,175,200,225,250,300,400,500 \mathrm{~m} / \mathrm{s}$.
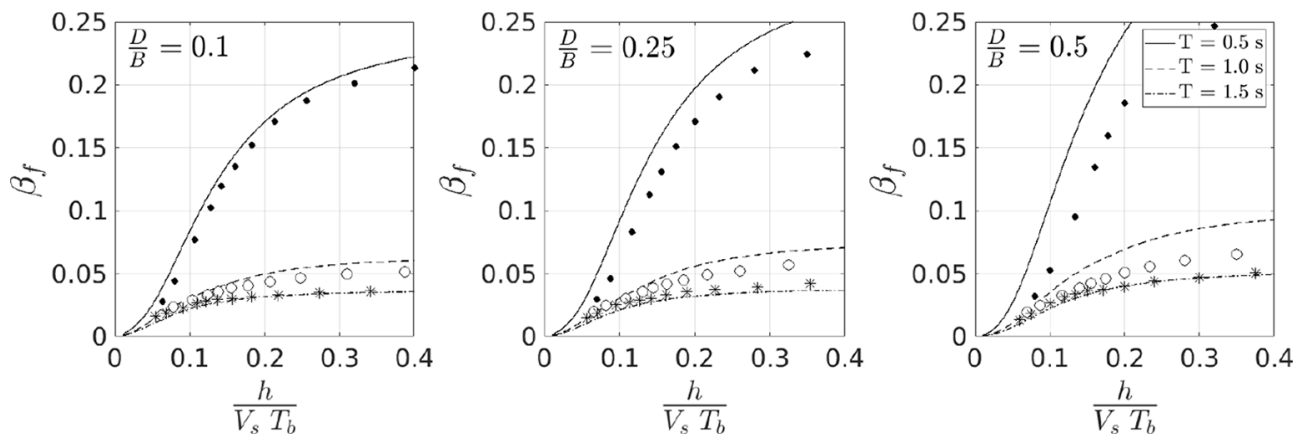

Figure 15. Identified foundation damping $\beta_{f}$ computed for soil properties $\rho_{s}=2000 \mathrm{~kg} / \mathrm{m}^{3}$ and $\nu_{s}=0.25$ using EnKI (markers) and NIST (lines) for building of fixed-base period $T=0.5,1.0,1.5 \mathrm{~s}$, first modal height $h=15,30,45 \mathrm{~m}$, foundation embedment $D=1.0,2.5,5.0 \mathrm{~m}$, and supported on 11 soils with shear velocity $V_{s}=80,100,125,150,175,200,225,250,300,400,500 \mathrm{~m} / \mathrm{s}$.

1. The estimated frequency of the interaction using the EnKI is different from the one obtained using the NIST recommendations.

2. The iterative method uses the first modal information to compute the lumped spring and dashpot coefficients while the EnKI-FIM estimates implicitly compensates for higher mode contribution since the soil parameters are estimated from the observations of the full FEM.

3. As discussed in the reduced order modeling section, we are explicitly considering the spring and dashpot coupling terms in the stiffness and damping matrices. It can be seen in both Equations 31 and 37 that mentioned coupling in the stiffness and damping matrices generate a slight increase in the period elongation ratio as well as a slight decrease in the radiation damping.

Regardless of the discrepancies presented, the global behavior of the two systems is very similar as shown in Figures 14 and 15. It is not a surprise that the period elongation ratios are well-captured given that the NIST recommendations compare well with the EnKI-FIM estimated lumped translational and rotational springs. Furthermore, the foundation damping for an equivalent one degree-of-freedom system is also well captured, which is expected 

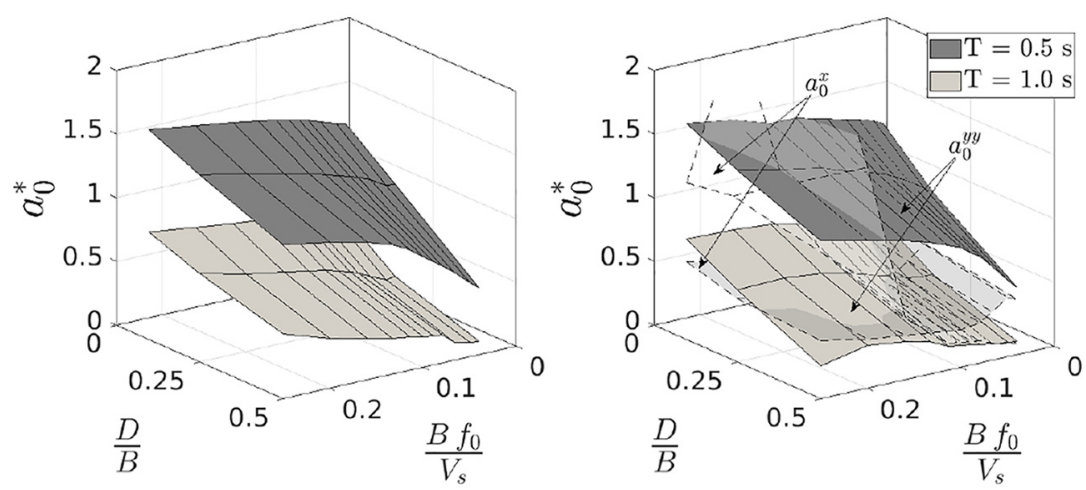

Figure 16. Optimal dimensionless frequency $a_{0}^{*}$ obtained using EnKI for (left) one dimensionless frequency and (right) for two dimensionless frequency.

since the contribution of the lumped rotational dashpot to the global foundation damping is minimal. These differences become more pronounced for the stiffer buildings, whose response has a strong rocking component ${ }^{3}$ that decreases the contribution of the foundation damping due to the coupling impedance term.

\section{Frequency estimation of optimal EnKI-FIM impedance}

Motivated by the inconsistencies that we demonstrated in the NIST definition of the most representative frequency to evaluate single valued stiffness and damping coefficients, we next used the EnKI algorithm to estimate the frequency at which one should read the numerically computed "true" impedance functions to determine the spring and dashpot values in the EnKI-FIM ROM. Again, we note that both the FEM and ROM analyses performed in this study are 2D plane-strain analyses. Therefore, all observed discrepancies are solely concerned with the extent to which the ROMs' impedance values are optimal. In this analysis, we consider two configurations: (a) only one frequency $a_{0}^{*}$ is considered for the lumped soil impedances and (b) two frequencies, one for the horizontal $\left(a_{0}^{x}\right)$ and one for the rotational $\left(a_{0}^{y y}\right)$ lumped soil impedances. The results of this analysis are shown in Figure 16. Figure 16 reveals two interesting results: the first confirms the hypothesis that the dimensionless frequency that controls the interaction is related to the rotational soil impedance. This can be seen since the dimensionless frequency $a_{0}^{*}$ in Figure 16a and $a_{0}^{\theta}$ in Figure 16b are very similar. The second result suggests that the optimal $a_{0}^{*}$ is not only a function of the building geometry and stiffness itself $(T)$, but also a function of the foundation embedment ratio and the signal frequency content. In fact, Conti et al. (2020) studied the seismic performance of bridge piers and concluded that the overall damping and natural frequency changes significantly with the layout of caisson and pile foundation. Therefore, improving the functional form of the dimensionless frequency $a_{0} \sim B / V_{s} \tilde{T}$ could potentially be achieved by including more parameters, such as $a_{0} \sim\left(h / V_{S} T\right),(h / B),(D / B),\left(B f_{0} / V s\right), \nu_{s}$. However, our set of results did not reveal a clear pattern on how this dependency could be taken into account. Our results did reveal, however, that evaluating the flexible-base period using the NIST recommended iterative method may result in less accurate results, especially when the soil is stiffer. Future work by the authors will focus on learning a parameterized functional form that can incorporate the characteristics of the structure, foundation and shaking frequency in the iterative 
approach of evaluating the global dynamic parameters of building structures accounting for SSI effects.

\section{Summary and conclusions}

The state-of-practice introduces a major simplification to transform the frequency domain analysis into a time domain analysis. It assumes that the frequency-dependent soil impedance functions can be replaced by single-valued functions evaluated at the flexible-base structure frequency. This simplification has been adopted to enable time history analyses using standard finite element packages. In this study, we found that despite the simplification outlined above, the NIST recommendations yield global dynamic behavior of building structures that compares well to direct finite element solutions. However, their performance deteriorates in capturing the response of the structure such as the floor displacement timeseries, and this is mainly due to the poor approximation of the lumped rotational dashpot coefficients. Consequently, the foundation of buildings with large period tends to be less dissipative since the radiation due to rocking is not properly estimated. The latter can be easily seen in Figure 9 for the lumped rotational component when $a_{0} \rightarrow 0$ which in particular occurs for flexible buildings and stiff soils. In this regard, results in Figure 12 show that the fixed-base period in general provides better results since $a_{0}(T)$ is greater than $a_{0}(\tilde{T})$ and thus the lumped rotational dashpot is better estimated; however, more test should be conducted to confirm the claim. Furthermore, the reader should be aware that the conditions in which NIST recommendations were tested are the best case scenario, in which the phase of the foundation input motion was also incorporated; thus, the discrepancies are only attributed to the frequency at which "true" impedance functions for plane strain setting are read.

Moreover, we showed that the foundation input motion effects are negligible for cases considered here with embedment ratios smaller than $D / B \leqslant 0.5$. The latter is due to the fact that foundation embedment are indeed small and vertically propagating shear waves do not create base slab averaging effects. Therefore, the free field motion can be directly applied without introducing substantial errors. Finally, we noticed that the selection of the dimensionless frequency $a_{0}$ that should be used to evaluate the impedance values depends on other dimensionless parameters, such as the frequency of the input signal and the embedment ratio. This suggests that determination of the representative $a_{0}$ is not a sole function of period elongation. In fact, Conti et al. (2020) showed that the effect on the SSI response should be referred to both the structure and the foundation system. Future work by the authors is aiming to learn a functional form that account for these parameters to estimate the most representative frequency of dynamic building-foundation-soil systems.

\section{Declaration of conflicting interests}

The author(s) declared no potential conflicts of interest with respect to the research, authorship, and/ or publication of this article.

\section{Funding}

The author(s) disclosed receipt of the following financial support for the research, authorship, and/ or publication of this article: The authors would like to acknowledge CONICYT-Becas Chile for the financial support to the first author for conducting doctoral studies at The California Institute of Technology. 


\section{ORCID iDs}

Danilo S Kusanovic (iD https://orcid.org/0000-0002-0935-2577

Elnaz Seylabi (D) https://orcid.org/0000-0003-0718-372X

Domniki Asimaki (D) https://orcid.org/0000-0002-3008-8088

\section{Notes}

1. The message passing interface (MPI) allows to exchange messages between multiple computers running a parallel program (single execution) across distributed memory.

2. Each point in the horizontal axis represents a building with fixed-base period $T$, embedment $D$, resting on a soil with shear wave velocity $V_{s}$. In each segment (delimited by black dashed lines) $V_{s}$ ranges from 80 to $500 \mathrm{~m} / \mathrm{s}$. For example, point 40 from left to right correspond to building with period $T=1.0 \mathrm{~s}$, embedment $D=1.0 \mathrm{~m}$, and shear wave velocity $V_{s}=225 \mathrm{~m} / \mathrm{s}$.

3. The rocking component for the short building is generated by the embedment which becomes more prominent as the embedment ratio increases.

\section{References}

Asimaki D, Garcia-Suarez J, Kusanovic D, Nguyen K and Seylabi EE (2019) Next generation reduced order models for soil-structure interaction. In: Earthquake geotechnical engineering for protection and development of environment and constructions: Proceedings of the 7th international conference on earthquake geotechnical engineering, ICEGE 2019, Rome, 17-20, June, p. 138. Boca Raton, FL: CRC Press.

Assimaki D (2004) Topography effects in the 1999 Athens earthquake: Engineering issues in seismology. PhD Thesis, Department of Civil and Environmental Engineering, Massachusetts Institute of Technology, Cambridge, MA.

Basu U and Chopra AK (2003) Perfectly matched layers for time-harmonic elastodynamics of unbounded domains: Theory and finite-element implementation. Computer Methods in Applied Mechanics and Engineering 192(11): 1337-1375.

Bathe K (2006) Finite Element Procedures. Prentice Hall. Available at: https://books.google.com/ books?id $=$ rWvefGICfO8C (accessed 9 December 2020).

Bielak J (1974) Dynamic behaviour of structures with embedded foundations. Earthquake Engineering \& Structural Dynamics 3(3): 259-274.

Bielak J and Jennings PC (1973) Dynamics of building-soil interaction. Bulletin of the Seismological Society of America 63(1): 9-48.

Broquedis F, Clet Ortega J, Moreaud S, Furmento N, Goglin B, Mercier G, Thibault S and Namyst R (2010) hwloc: A generic framework for managing hardware affinities in HPC applications. In: 18th Euromicro conference on parallel, distributed and network-based processing, Pisa, 17-19 February.

Conti R, Di Laora R, Licata V, Iovino M and de Sanctis L (2020) Seismic performance of bridge piers: Caisson vs pile foundations. Soil Dynamics and Earthquake Engineering 130: 105985. Available at: http://www.sciencedirect.com/science/article/pii/S0267726119309200 (accessed 9 December 2020).

Conti R, Morigi M, Rovithis E, Theodoulidis N and Karakostas C (2018) Filtering action of embedded massive foundations: New analytical expressions and evidence from 2 instrumented buildings. Earthquake Engineering \& Structural Dynamics 47(5): 1229-1249.

Cook RD, Malkus DS, Plesha ME and Witt RJ (2007) Concepts and Applications of Finite Element Analysis. Hoboken, NJ: John Wiley.

Day S (1978) Seismic Response of Embedded Foundations. American Society of Civil Engineers. Available at: https://books.google.com/books?id=Uni2HAAACAAJ (accessed 9 December 2020).

Evensen G (2006) Data Assimilation: The Ensemble Kalman Filter. Berlin: Springer-Verlag. 
Gabriel E, Fagg GE, Bosilca G, Angskun T, Dongarra JJ, Squyres JM, Sahay V, Kambadur P, Barrett B, Lumsdaine A, Castain RH, Daniel DJ, Graham RL and Woodall TS (2004) Open MPI: Goals, concept, and design of a next generation MPI implementation. In: Proceedings of the 11th European PVM/MPI users' group meeting, Budapest, pp. 97-104. Available at: https:// www.open-mpi.org/papers/euro-pvmmpi-2004-overview/euro-pvmmpi-2004-overview.pdf (accessed 9 December 2020).

Gazetas G (1991) Foundation Vibrations. Boston, MA: Springer.

Gazetas G (1996) Soil Dynamics and Earthquake Engineering-Case Studies. Athens, Greece: Simeon Publications.

Givens MJ, Mylonakis G and Stewart JP (2016) Modular analytical solutions for foundation damping in soil-structure interaction applications. Earthquake Spectra 32(3): 1749-1768.

Guyan RJ (1965) Reduction of stiffness and mass matrices. AIAA Journal 3(2): 380-380.

Hughes T (2012) The Finite Element Method: Linear Static and Dynamic Finite Element Analysis. Dover Civil and Mechanical Engineering and Dover Publications. Available at: https:// books.google.com/books?id = cHH2n_qBK0IC (accessed 9 December 2020).

Hursey J, Mallove E, Squyres JM and Lumsdaine A (2007) An Extensible Framework for Distributed Testing of MPI Implementations. In: Cappello F, Herault T and Dongarra J (eds) Recent Advances in Parallel Virtual Machine and Message Passing Interface. EuroPVM/MPI. Lecture Notes in Computer Science, vol 4757. Berlin, Heidelberg: Springer.

Iglesias MA, Law KJH and Stuart AM (2013) Ensemble Kalman methods for inverse problems. Inverse Problems 29(4): 045001.

Irons B (1965) Structural eigenvalue problems: Elimination of unwanted variables. AIAA Journal 3(5): 961-962.

Kausel E, Whitman RV, Morray JP and Elsabee F (1978) The spring method for embedded foundations. Nuclear Engineering and Design 48(2): 377-392. Available at: http: //www.sciencedirect.com/science/article/pii/0029549378900857 (accessed 9 December 2020).

Kucukcoban S and Kallivokas L (2011) Mixed perfectly-matched-layers for direct transient analysis in $2 \mathrm{~d}$ elastic heterogeneous media. Computer Methods in Applied Mechanics and Engineering 200(1): 57-76. Available at: http://www.sciencedirect.com/science/article/pii/S0045782510002252 (accessed 9 December 2020).

Kusanovic D, Seylabi E and Asimaki D (2018) System identification of soil-structure interaction mechanisms for building structures. In: SMIP18 seminar on utilization of strong-motion data, pp. 53-68. Sacramento, CA: SMIP18.

Kusanovic D, Seylabi E, Kottke A and Asimaki D (2020) Seismo-VLab: A parallel object-oriented platform for reliable nonlinear seismic wave propagation and soil-structure interaction simulation. Computer Methods in Applied Mechanics and Engineering.

Law K, Stuart A and Zygalakis K (2016) Data Assimilation: A Mathematical Introduction (Texts in Applied Mathematics). Cham: Springer.

Lysmer $\mathbf{J}$ and Kuhlemeyer RL, Institute of Transportation and Traffic Engineering, Soil Mechanics and Bituminous Materials Research Laboratory, University of California, Berkeley, Geotechnical Engineering Group (1969) Finite Dynamic Model for Infinite Media. Berkeley, CA: Department of Civil Engineering, University of California, Institute of Transportation and Traffic Engineering, Soil Mechanics Laboratory.

Maravas A, Mylonakis G and Karabalis DL (2014) Simplified discrete systems for dynamic analysis of structures on footings and piles. Soil Dynamics and Earthquake Engineering 61-62: 29-39. Available at: http://www.sciencedirect.com/science/article/pii/S0267726114000177 (accessed 9 December 2020).

Mynolakis G and Gazetas G (2000) Seismic soil-structure interaction: Beneficial or detrimental? Journal of Earthquake Engineering 4(3): 277-301.

Pais A and Kausel E (1988) Approximate formulas for dynamic stiffnesses of rigid foundations. Soil Dynamics and Earthquake Engineering 7(4): 213-227. Available at: http: //www.sciencedirect.com/science/article/pii/S0267726188800058 (accessed 9 December 2020).

Parmelee RA (1967) Building-foundation interaction effects. Journal of the Engineering Mechanics Division 93(2): 131-152. 
Seylabi E (2016) Reduced order modeling of soil structure interaction problems. PhD Thesis, University of California, Los Angeles, Los Angeles, CA.

Seylabi E, Jeong C and Taciroglu E (2016) On numerical computation of impedance functions for rigid soil-structure interfaces embedded in heterogeneous half-spaces. Computers and Geotechnics 72: $15-27$.

Stewart JP, Crouse C, Hutchinson TC, Lizundia B, Naeim F and Ostadan F (2012) Soil-Structure Interaction for Building Structures (Grant/Contract Reports (NISTGCR) - 12-917-21). Gaithersburg, MD: National Institute of Standards and Technology, U.S. Department of Commerce.

Stewart JP, Fenves GL and Seed RB (1999a) Seismic soil-structure interaction in buildings. I: Analytical methods. Journal of Geotechnical and Geoenvironmental Engineering 125(1): 26-37.

Stewart JP, Seed RB and Fenves GL (1999b) Seismic soil-structure interaction in buildings. II: Empirical findings. Journal of Geotechnical and Geoenvironmental Engineering 125(1): 38-48.

Veletsos AS and Meek JW (1974) Dynamic behaviour of building-foundation systems. Earthquake Engineering \& Structural Dynamics 3(2): 121-138.

Veletsos AS and Nair V (1975) Seismic interaction of structures on hysteretic foundations. ASCE Journal of the Structural Division 101: 109-129.

Wolf JP (1985) Dynamic Soil-Structure Interaction (Prentice-Hall International Series in Civil Engineering and Engineering Mechanics). Prentice-Hall. Available at: https://books.google.com/ books?id =0ORRAAAAMAAJ (accessed 9 December 2020).

Wolf JP (1989) Soil-structure-interaction analysis in time domain. Nuclear Engineering and Design 111(3): 381-393. Available at: http://www.sciencedirect.com/science/article/pii/0029549389902495 (accessed 9 December 2020).

Wolf JP and Obernhuber P (1985) Non-linear soil-structure-interaction analysis using dynamic stiffness or flexibility of soil in the time domain. Earthquake Engineering \& Structural Dynamics 13(2): 195-212.

Zania V (2014) Natural vibration frequency and damping of slender structures founded on monopiles. Soil Dynamics and Earthquake Engineering 59: 8-20. Available at: http: //www.sciencedirect.com/science/article/pii/S0267726114000086 (accessed 9 December 2020).

\section{Appendix I}

\section{Period elongation and radiation damping derivations}

Let us consider two different systems as depicted in Figure 17.

The first system in Figure 17a has one floor with height $\bar{h}=h+D$, stiffness $k \in \mathbb{R}^{+}$, and structural damping $\beta_{i} \in \mathbb{R}^{+}$. The second systems in Figure $17 \mathrm{~b}$ are a fixed-base singledegree-of-freedom system with mass $m \in \mathbb{R}^{+}$, modified-stiffness $\tilde{k} \in \mathbb{R}^{+}$, modifiedfundamental period $\tilde{T} \in \mathbb{R}^{+}$, and modified damping $\beta_{f} \in \mathbb{R}^{+}$. These systems are presented in detail in Stewart et al. (1999a) and Givens et al. (2016).

In the case of the equivalent fixed-base system, the total static displacement $\tilde{\Delta}$ generated by an applied external load $F$ is computed as

$$
\tilde{\Delta}=\frac{F}{\tilde{k}} \text {, with } \tilde{\Delta} \in \mathbb{R},
$$

The total displacement of the flexible-base system when an external load $F$ is applied is computed as: 


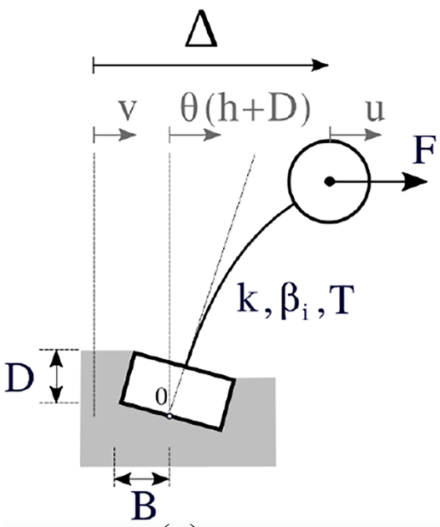

(a)

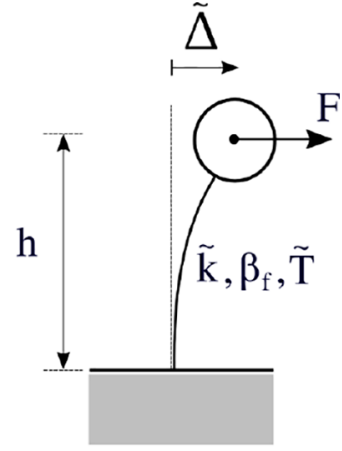

(b)

Figure 17. Reduction from (a) the simplified flexible-base system into (b) the equivalent fixed-base system.

$$
\begin{aligned}
\Delta & =u+v+\theta \bar{h} \\
& =\frac{F}{k}+\frac{F\left(k_{y y}-\bar{h} k_{x y}\right)}{k_{y y} k_{x}-k_{x y}^{2}}+\frac{F\left(k_{x y}-\bar{h} k_{x}\right)}{k_{x y}^{2}-k_{x} k_{y y}} \bar{h}^{2} \\
& =F\left(\frac{1}{k}+\frac{\bar{h}^{2} k_{x}-2 \bar{h} k_{x y}+k_{y y}}{k_{x} k_{y y}-k_{x y}^{2}}\right) .
\end{aligned}
$$

Note that, for the fixed-base system to be equivalent to the flexible-base system, the displacements must be equal, that is, $\tilde{\Delta}=\Delta$. By comparison of Equations 23 and 24, we conclude that the following relation between the stiffness of the two systems must hold

$$
\frac{1}{\tilde{k}}=\frac{1}{k}+\frac{\bar{h}^{2} k_{x}-2 \bar{h} k_{x y}+k_{y y}}{k_{x} k_{y y}-k_{x y}^{2}} .
$$

Next, we now represent the impedance $\mathbb{K} \in \mathbb{C}$ of the system in complex format, this is

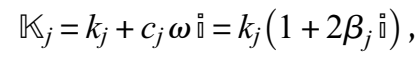

where $\beta_{j}=\omega c_{j} / 2 k_{j}$ and the index $j$ represents the horizontal, vertical, or coupled degree of freedom to be considered. Employing Equation 25 and the impedance represented in complex format, one can write

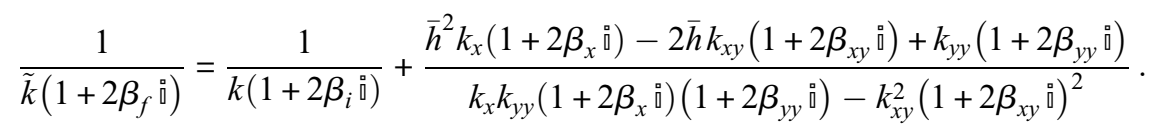

If each term in the previous expression is multiplied by its complex conjugated and assuming that the damping ratio for all degree of freedom are small so that $\left(\beta_{j}\right)^{2} \approx 0$, then Equation 27 becomes

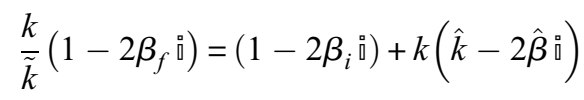


where the variables $\hat{k} \in \mathbb{R}^{+}$and $\hat{\beta} \in \mathbb{R}^{+}$in Equation 28 are defined as follows

$$
\begin{gathered}
\hat{k}=\frac{\bar{h}^{2} k_{x}-2 \bar{h} k_{x y}+k_{y y}}{k_{x} k_{y y}-k_{x y}^{2}} \\
\hat{\beta}=\frac{k_{x}\left(k_{y y}-\bar{h} k_{x y}\right)^{2} \beta_{x}-2 k_{x y}\left(\bar{h} k_{x}-k_{x y}\right)\left(\bar{h} k_{x y}-k_{y y}\right) \beta_{x y}+k_{y y}\left(k_{x y}-\bar{h} k_{x}\right)^{2} \beta_{y y}}{\left(k_{x} k_{y y}-k_{x y}^{2}\right)^{2}}
\end{gathered}
$$

Solving for the real component of Equation 28 yields

$$
\frac{\tilde{T}}{T}=\sqrt{1+\frac{k}{k_{x} k_{y y}-k_{x y}^{2}}\left(\bar{h}^{2} k_{x}-2 \bar{h} k_{x y}+k_{y y}\right)} .
$$

Solving for the imaginary component of Equation 28 yields

$$
\begin{aligned}
\beta_{f}= & \frac{\tilde{k}}{k} \beta_{i}+\frac{\tilde{k}}{k}\left(\frac{k_{x}\left(k_{y y}-\bar{h} k_{x y}\right)^{2}}{\left(k_{x} k_{y y}-k_{x y}^{2}\right)^{2}} k \beta_{x}-2 \frac{k_{x y}\left(\bar{h} k_{x}-k_{x y}\right)\left(\bar{h} k_{x y}-k_{y y}\right)}{\left(k_{x} k_{y y}-k_{x y}^{2}\right)^{2}} k \beta_{x y}+\right. \\
& \left.\frac{k_{y y}\left(k_{x y}-h k_{x}\right)^{2}}{\left(k_{x} k_{y y}-k_{x y}^{2}\right)^{2}} k \beta_{y y}\right) \\
= & \frac{\tilde{k}}{k} \beta_{i}+\frac{\tilde{k}}{k}\left(\frac{k}{k_{x}} \beta_{x}\left(\frac{1-\bar{h} \frac{k_{x y}}{k_{y y}}}{1-\frac{k_{x y}^{2}}{k_{x} k_{y y}}}\right)^{2}-2 \frac{\bar{h} k k_{x y}}{k_{x} k_{y y}} \beta_{x y} \frac{\left(1-\frac{k_{x y}}{h k_{x}}\right)\left(\bar{h} \frac{k_{x y}}{k_{y y}}-1\right)}{\left(1-\frac{k_{x y}^{2}}{k_{x} k_{y y}}\right)^{2}}+\right. \\
& \left.\frac{k \bar{h}^{2}}{k_{y y}} \beta_{y y}\left(\frac{1-\frac{k_{x y}}{h k_{x}}}{1-\frac{k_{x y}^{2}}{k_{x} k_{y y}}}\right)^{2}\right)
\end{aligned}
$$

Using the translational period and the rocking period definitions, the following identities can be written

$$
\begin{aligned}
\frac{\tilde{k}}{k} & =\frac{\tilde{k}}{m} \frac{m}{k}=\frac{\tilde{\omega}_{n}^{2}}{\omega_{n}^{2}}=\frac{1}{\left(\frac{\tilde{T}}{T}\right)^{2}}, \\
\frac{k}{k_{x}} & =\frac{k}{m} \frac{m}{k_{x}}=\frac{\omega_{n}^{2}}{\omega_{x}^{2}}=\frac{1}{\left(\frac{T}{T_{x}}\right)^{2}}, \\
\frac{k \bar{h}^{2}}{k_{y y}} & =\frac{k}{m} \frac{m \bar{h}^{2}}{k_{y y}}=\frac{\omega_{n}^{2}}{\omega_{\theta}^{2}}=\frac{1}{\left(\frac{T}{T_{\theta}}\right)^{2}} \\
\frac{k \bar{h}}{\sqrt{k_{x} k_{y y}}} & =\sqrt{\frac{k}{k_{x}}} \sqrt{\frac{k \bar{h}^{2}}{k_{\theta}}}=\frac{1}{\left(\frac{T}{T_{x}}\right)} \frac{1}{\left(\frac{T}{T_{\theta}}\right)} .
\end{aligned}
$$


Replacing the later expressions yields

$$
\begin{aligned}
\beta_{f}= & \frac{\beta_{i}}{\left(\frac{\tilde{T}}{T}\right)^{2}}+\frac{1}{\left(\frac{\tilde{T}}{T}\right)^{2}}\left(\frac{\beta_{x}}{\left(\frac{T}{T_{x}}\right)^{2}}\left(\frac{1-\bar{h} \frac{k_{x y}}{k_{y y}}}{1-\frac{k_{x y}^{2}}{k_{x} k_{y y}}}\right)^{2}+\frac{\beta_{y y}}{\left(\frac{T}{T_{\theta}}\right)^{2}}\left(\frac{1-\frac{k_{x y}}{h k_{x}}}{1-\frac{k_{x y}^{2}}{k_{x} k_{y y}}}\right)^{2}-\right. \\
& \left.\frac{2}{\left(\frac{T}{T_{x}}\right)} \frac{\beta_{x y}}{\left(\frac{T}{T_{\theta}}\right)} \frac{k_{x y}}{\sqrt{k_{x} k_{y y}}} \frac{\left(1-\frac{k_{x y}}{h k_{x}}\right)\left(\bar{h} \frac{k_{x y}}{k_{y y}}-1\right)}{\left(1-\frac{k_{x y}^{2}}{k_{x} k_{y y}}\right)^{2}}\right) .
\end{aligned}
$$

Now, if each term in Equation 25 is first represented in complex format, and then is multiplied by its complex conjugate assuming that the damping ratio for all degree of freedom are small so that $\left(\beta_{j}\right)^{2} \approx 0$, then one can solve for the imaginary component and obtain the reduced foundation-damping provided in Equation 37,

$$
\begin{aligned}
\beta_{f}= & \frac{\beta_{i}}{\left(\frac{\tilde{T}}{T}\right)^{2}}+\frac{\beta_{x}}{\left(\frac{\tilde{T}}{T_{x}}\right)^{2}}\left(\frac{1-\bar{h} \frac{k_{x y}}{k_{y y}}}{1-\frac{k_{x y}^{2}}{k_{x} k_{y y}}}\right)^{2}+\frac{\beta_{y y}}{\left(\frac{\tilde{T}}{T_{\theta}}\right)^{2}}\left(\frac{1-\frac{k_{x y}}{h k_{x}}}{1-\frac{k_{x y}^{2}}{k_{x} k_{y y}}}\right)^{2}- \\
& \frac{2}{\left(\frac{\tilde{T}}{T_{x}}\right)} \frac{\beta_{x y}}{\left(\frac{\tilde{T}}{T_{\theta}}\right)} \frac{k_{x y}}{\sqrt{k_{x} k_{y y}}} \frac{\left(1-\frac{k_{x y}}{h k_{x}}\right)\left(\bar{h} \frac{k_{x y}}{k_{y y}}-1\right)}{\left(1-\frac{k_{x y}^{2}}{k_{x} k_{y y}}\right)^{2}} .
\end{aligned}
$$

Remark. Note that in both Equations 31 and 37 when the stiffness $k_{x y}=0$, the expression reduces to the one presented in Bielak and Jennings (1973), Veletsos and Meek (1974), Stewart et al. (1999a), and Givens et al. (2016). Also, if the contribution of the secondorder damping terms are neglected (i.e. $\beta_{i} \beta_{j} \sim 0$ ) in Zania (2014), then the result coincides once again with Equations 31 and 37.

\section{Ensemble Kalman inversion methodology for SSI}

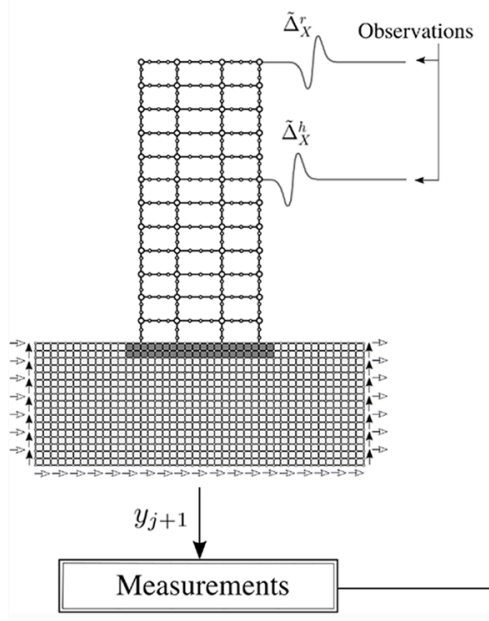

(a)

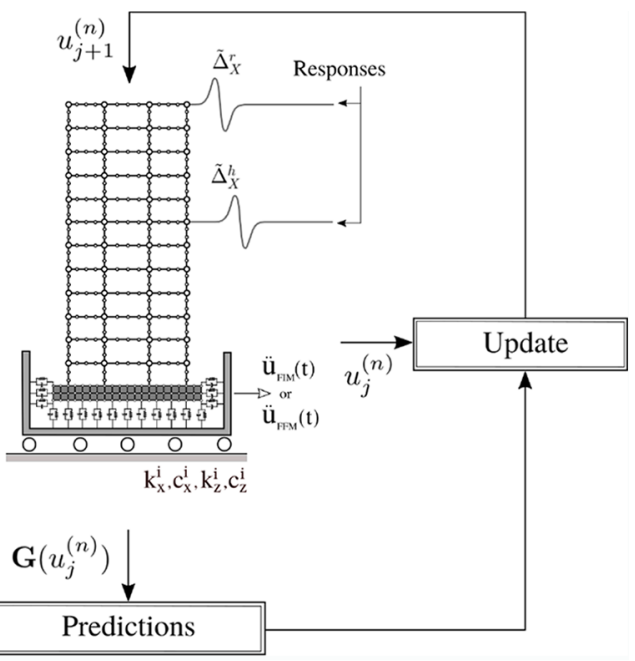

(b)

Figure 18. Parameter estimation framework based on EnKI applied to SSI (a) full finite element model from which the "true" responses are computed (observations) and (b) reduced order method from which the spring and dashpot coefficients are estimated. 